\title{
Environmentally conscious logistics planning for food grain industry considering wastages employing multi objective hybrid particle swarm optimization
}

\begin{abstract}
In this paper, a hub and spoke network based multi-objective green transportation model is developed while evaluating optimal shipment quantity, modal choice, route selection, hub location, and vehicle velocity decisions in Indian food grain context. A hybrid version of multiobjective meta-heuristic, Multi-Objective Particle Swarm Optimization with Differential Evolution (MOPSODE) is proposed to tackle the resulting non-linear formulation. Benchmarking with NSGA-II confirms the dominance of MOPSODE over NSGAII pertaining to near optimal pareto fronts obtained for the tested cases. Finally, the study derives the economic and environmental impact of varying hub location level, food grain wastage threshold and intermodal hub capacity.
\end{abstract}

Keywords: Food grain transportation; GHG emission reduction; Sustainability; Multi-objective optimization; MOPSODE; NSGA II

\section{Introduction}

According to Netherlands Environment Assessment Agency, the GHG emissions reduction in different countries ranged between $1.3 \%$ to $6 \%$, whereas India and Indonesia have registered 4.7\% and 6.4\% increase in their GHG emissions (Olivier et al., 2017). Thirty eight percent of global GHG emissions is attributed to transportation and agriculture sectors (IPCC 2014). Owing to large scale of food grain transportation operations in developing and densely populated economies, holistic design of food grain systems demand deeper attention of transportation research. To this end, this paper proposes a cost effective sustainable intermodal transportation model to facilitate environmental friendly food grain shipments considering GHG emissions in Indian context. 
Food and Agriculture Organization (FAO) estimates that only two-thirds of the total produce manage to reach the consumer's table. FAO points out that a kilogram of food lost in the later stages of supply chain (post harvesting stages) possess higher carbon intensity than at preliminary stages. In India, agriculture contributes to $18 \%$ of total GHG emissions (INCCA 2010). The forecasts by Indian Council of Agricultural Research (ICAR) suggest that the areaaveraged annual mean warming is estimated to $3{ }^{\circ} \mathrm{C}$ by 2050 . Thus, there is an immense need for the design and implementation of cost effective and carbon sensitive food grain supply networks in Indian context considering food grain wastages.

The large network of food grain supply chain considered in this paper is geographically widespread consisting of storage facilities, intermodal hubs connected by rail and road links to facilitate the transport between surplus and deficit states. Streamlining of transportation operations in airline, shipping, telecommunication and large retail delivery networks has been achieved by the implementation of hub and spoke framework. According to Meng and Wang (2011) the consolidation at intermodal hubs reap significant cost savings for large networks from economies of scale. Hence, this paper attempts to model the food grain transportation problem on a hub and spoke framework as different from the traditional point to point network. A multi-objective cost minimization model for the interstate transportation of food grains considering cost and GHG emission (total grams of carbon dioxide equivalents) as two separate objectives is formulated. The first objective minimizes the total transportation, hub location, and service time violation costs whereas the second objective minimizes the cost of emissions resulting from transportation and intermodal hub facilities. The mathematical model is designed to fulfill a multi period deterministic demand while observing wastages at intermodal facilities. The optimal choice of mode in addition to other decisions including shipment quantity allocation, facility allocation and vehicle speed is made while balancing the tradeoff between the first and second objectives. The multi-objective problem is contingent with respect 
to warehouse capacity, intermodal transfer capacity, maximum allowable food grain wastage, flow balance, vehicle capacity and speed restrictions. The primary question that this research aims to address is, "In what way does the modelling for wastages at intermodal hubs with simultaneous consideration to supply network cost and GHG emissions in food grain transportation network design influence itself ?'. In this regard, this paper attempts to answer the following specific important questions:

- What is the optimal food grain shipment quantity and shipment route?

- What is the optimal intermodal hub allocation plan?

- What is the optimal choice of transportation mode inside the origin and destination states?

- What are the optimal speeds of rail and road transport vehicle that minimize GHG emissions without violating service time restrictions?

- How does the variation in intermodal hub location level, maximum food grain wastage threshold and intermodal hub capacity, effect the total supply network cost and total GHG emission?

The remainder of the paper is organized as follows. The next section outlines the literature review conducted in the context of this work. Section 3 presents the problem description. The mathematical model with detailed description of indices, sets, parameters, decision variables, objective function and constraints are described in section 4. Section 5 describes the multi objective solution methodology proposed to solve the given food grain transportation problem. Section 6 summarizes the plan of experiments and data used for further study. Results from computational validation, benchmarking and sensitivity analysis with development of problem specific insights are reported in section 7. Finally, section 8 presents conclusion and future work. 


\section{Literature review}

The significant contribution from transportation sector towards total supply network GHG emissions has attracted the critical focus of researchers and practitioners to deal with green supply chain network design problem (Centobelli et al. 2017). Table 1 shows that most studies apply optimization models based on mixed integer linear or non-linear programming (MILP or MINLP) and non-linear programming (NLP). Only two exceptional papers present simulation models (Harris et al., 2011; van der Vorst et al., 2009). All reviewed studies take into account transportation costs, while only about every second study considers hub or facility location costs. Vehicle speed decisions are hardly addressed, only Harris et al. (2011), De et al. (2017) and Kumar et al. (2016) consider this factor, and wastages are completely ignored in the reviewed studies. Chang and Morlok (2005) proved the economic advantage of maintaining a constant speed profile in land transport with level tangent paths. However, research that encapsulates vehicle speed decisions with major supply network decisions such as transportation flow, facility location, modal choices while simultaneously minimizing total supply network costs and emissions are meagerly found in literature and is still in its infancy. Transportation modes are addressed as multimodal (Zahiri et al., 2017; Liotta et al., 2015; Fattahi and Govindan, 2018) or intermodal (Maiyar and Thakkar, 2018), but most papers omit distinguishing between different modes. Most of the studies address vehicle hub capacities while very few studies are found to explore p-hub median constraints. Service-time violation constraints are addressed in five different studies while a higher number of studies formulated multi-period models. There is limited research that addresses sustainability issues in food supply chains (Pathak et al., 2010; Wakeland et al., 2012; Garnett, 2011). Real case studies pertaining to different countries reiterate the need for sustainability in food grain supply chains (O’Donnell et al., 2009, Maiyar and Thakkar, 2017). To the best of our knowledge, there is lack of studies that capture wastages across intermodal hubs while dealing with environmental 
sustainability in food grain supply chain context. This, paper attempts to address this gap by focusing on intermodal hub location decisions subject to wastages, modal choices and transportation flow decisions in the presence of fixed vehicle speed decisions with focus to reduce total GHG emissions in Indian food grain supply chain.

Multi-objective problems have been addressed by exact as well as meta-heuristic approaches. Dual lexicographic max-min (LMM) maximization, hybrid k-means and Tukeys statistical method, long range Lagrangian and Eulerian transport methods are some exact optimization approaches employed to solve green transportation problems (Niknamfar and Niaki, 2016; Velázquez-Martínez et al., 2016; Sundarakani et al., 2010). Multi-objective metaheuristic optimization was pioneered by Deb et al. (2002) with their introduction of non-dominated sorting genetic algorithm (NSGA-II) to solve combinatorial and large scale NP-hard problems. In subsequent years several authors have proposed novel multi-objective metaheuristics by extending single objective versions of particle swarm optimization, genetic algorithm, tabu search, variable neighborhood search, simulated annealing and chemical reaction optimization (Vahdani and Zandieh 2010, Li and Li. 2015, Mogale et al. 2018). Particle swarm optimization based metaheuristics are well known global minimizers and are highly promising for generating solutions in the close vicinity of global optimum (Epitropakis et al. 2012). The global optimality of its multi-objective variant MOPSDE was validated by benchmarking with NSGA-II algorithm by Su and Chi (2017) for different problems. Considering the case of evolutionary metaheuristic optimization, Hanne (1999) concludes that although the global optimality condition is theoretically not proven, in practice, these algorithms may perform much better than the ones whose global convergence can be proven. Therefore, it is more 
Table 1. Comparative study of relevant literature that address environment sustainability with present work

\begin{tabular}{|c|c|c|c|c|c|c|c|c|c|c|}
\hline Study & $\begin{array}{l}\text { Transportation } \\
\text { cost }\end{array}$ & $\begin{array}{l}\text { Hub/Facility } \\
\text { location cost }\end{array}$ & $\begin{array}{l}\text { Vehicle speed } \\
\text { decision }\end{array}$ & Wastages & $\begin{array}{l}\text { Service/lead } \\
\text { time restriction }\end{array}$ & $\begin{array}{l}\text { p-hubs } \\
\text { constraint }\end{array}$ & $\begin{array}{l}\text { Intermodal } \\
\text { /multimodal }\end{array}$ & $\begin{array}{l}\text { Vehicle/hub } \\
\text { capacity }\end{array}$ & $\begin{array}{l}\text { Multi } \\
\text { period }\end{array}$ & Model \\
\hline $\begin{array}{l}\text { Zhalechian et al. } \\
\text { (2016) }\end{array}$ & $\checkmark$ & $\checkmark$ & $x$ & $x$ & $x$ & $x$ & $x$ & $\checkmark$ & $\checkmark$ & MINLP* \\
\hline Zahiri et al. (2017) & $\checkmark$ & $\checkmark$ & $x$ & $x$ & $\checkmark$ & $x$ & MM & $\checkmark$ & $\checkmark$ & MILP* \\
\hline $\begin{array}{l}\text { Chavez et al. } \\
(2018)\end{array}$ & $\checkmark$ & $\checkmark$ & $x$ & $x$ & $x$ & $x$ & $x$ & $\checkmark$ & $\checkmark$ & MILP \\
\hline Liotta et al. (2015) & $\checkmark$ & $x$ & $x$ & $x$ & $\checkmark$ & $x$ & $\mathrm{MM}$ & $\checkmark$ & $x$ & MILP \\
\hline Wang et al. (2011) & $\checkmark$ & $\checkmark$ & $x$ & $x$ & $x$ & $x$ & $x$ & $\checkmark$ & $x$ & MILP \\
\hline $\begin{array}{l}\text { Fattahi and } \\
\text { Govindan (2018) }\end{array}$ & $\checkmark$ & $\checkmark$ & $x$ & $x$ & $x$ & $x$ & $\mathrm{MM}$ & $\checkmark$ & $\checkmark$ & MILP \\
\hline Harris et al. (2011) & $\checkmark$ & $x$ & $\checkmark$ & $x$ & $x$ & $x$ & $x$ & $x$ & $x$ & Simulation \\
\hline $\begin{array}{l}\text { Maiyar and } \\
\text { Thakkar (2018) }\end{array}$ & $\checkmark$ & $\checkmark$ & $x$ & $x$ & $x$ & $\checkmark$ & IM & $\checkmark$ & $\checkmark$ & MINLP \\
\hline De et al. (2017) & $\checkmark$ & $x$ & $\checkmark$ & $x$ & $\checkmark$ & $x$ & $x$ & $\checkmark$ & $\checkmark$ & MINLP \\
\hline Kumar et al. (2016) & $\checkmark$ & $x$ & $\checkmark$ & $x$ & $\checkmark$ & $x$ & $x$ & $\checkmark$ & $\checkmark$ & MINLP \\
\hline $\begin{array}{l}\text { van der Vorst et al. } \\
(2009)\end{array}$ & $\checkmark$ & $x$ & $x$ & $x$ & $\checkmark$ & $x$ & $x$ & $\checkmark$ & $x$ & Simulation \\
\hline $\begin{array}{l}\text { Sahebjamnia et al. } \\
\text { (2018) }\end{array}$ & $\checkmark$ & $\checkmark$ & $x$ & $x$ & $x$ & $x$ & $x$ & $\checkmark$ & $x$ & MILP \\
\hline Saberi (2018) & $\checkmark$ & $x$ & $x$ & $x$ & $x$ & $x$ & $x$ & $x$ & $\checkmark$ & $\mathrm{NLP}^{*}$ \\
\hline Lee et al. (2010) & $\checkmark$ & $\checkmark$ & $x$ & $x$ & $x$ & $x$ & $x$ & $\checkmark$ & $x$ & MILP \\
\hline Present study & $\checkmark$ & $\checkmark$ & $\checkmark$ & $\checkmark$ & $\checkmark$ & $\checkmark$ & $\mathrm{MM}$ & $\checkmark$ & $\checkmark$ & MINLP \\
\hline
\end{tabular}

${ }^{*}$ NLP- Non-linear problem, MILP- Mixed integer linear problem, MINLP- Mixed integer nonlinear problem, IM- Intermodal, MM- Multimodal 
important that the performance of the algorithm is verified through benchmarking with standard meta-heuristic counterparts which is an effective surrogate to validate the global optimality, especially for real and complex problems as in the present case. This paper extends the multi-objective algorithm proposed by Su and Chi, (2017) by incorporating constraint violation based penalty allocation scheme for achieving faster convergence rates.

A critical review of the aforementioned literature reveals that there is an immense need of integrated models that simultaneously capture wastages in synergy with transportation, hub location and vehicle speed decisions for minimizing the overall cost with specific focus of reducing GHG emission in food grain supply chain. The underpinning contributions of this paper are three fold. Firstly, a multi-objective mathematical model is developed to analyze the trade-off between total supply network costs and total GHG emissions in the presence of modal choices and food grain wastages considering Indian food grain supply chain context. The first objective minimizes the total supply network costs which includes total transportation cost, total hub location cost, and total service time violation cost, whereas, the second objective minimizes the total GHG emissions emitted through transportation of vehicles (rail and road) and food grain wastages due to handling at facilities. Therefore the main contribution in this particular part of the study is to develop an environmentally conscious wastage aware and economically convenient multi-objective mathematical model for a food grain transportation network with intermodal as well as multi-modal setups while incorporating decisions pertaining to movement quantity, movement route, modal choice and vehicle speeds. The second contribution of this study lies in devising a self-tailored version of multi-objective particle swarm optimization algorithm which includes a new penalty allocation scheme for dealing with constraint violations. Thirdly, a hub closure impact index is proposed to capture the economic impact of closing intermodal hubs on the economic and environmental objectives. 


\section{Problem environment}

As discussed earlier, the transportation problem addressed in this paper is inspired from Indian food grain supply chain network that connects two states, Andhra Pradesh and Tamil Nadu, belonging from Southern part of the country. The states are geographically divided in to finite number of regions. The food grains to be transported from one state to the other (inter-state shipment) are lifted from central pool stock stored in the warehouses owned by Food Corporation of India (FCI). Each region consists of a finite number of warehouses. A fixed number of such warehouses are designated as potential intermodal transfer hubs from where the incoming flow of food grains from trucks are transferred to trains. Thus, the food grain warehouses present in each region are categorized in to two types: (1) Intermodal hub warehouses and (2) Non-hub warehouses. Intermodal hub warehouses are the warehouses which are capable of facilitating intermodal transfer operations, whereas non-hub warehouses are the ones that are used for only storing the food grains and do not handle intermodal transfers. The flow of food grains either originate from a non-hub warehouse or from an intermodal hub warehouse of the surplus (origin) state and continues to reach the designated non-hub warehouse or intermodal hub warehouse of the deficit (destination) state passing through the origin and destination intermodal hub warehouses. The two types of warehouses are allowed to be connected either by road or rail. Fig. 1 displays the structure of food grain supply network chosen for addressing the green transportation problem with the multimodal links, connecting distinct nodal elements such as hub and non-hub warehouses situated within the regions of the origin and destination states.

Significant portion of the food grains is lost while handling at intermodal hubs. In this paper, the food grain wastages are quantitatively captured by estimating the amount of food grains lost as a fixed proportion of the total quantity handled at the transfer facility. The proportion of food grains wasted varies with respect to each facility and is denoted by $\mu_{o k}$ and $\mu_{d m}$, where 
$k \in H_{o}$, and $m \in H_{d} . H_{o}$ and $H_{d}$ are the set of potential hubs in origin and destination states respectively. Fig. 2 illustrates the transfer logistics associated with shipment quantity from origin to destination warehouse through the intermodal hubs considering food grain wastages for a simple example of three origin and three destination intermodal hubs. Imagining that 100 units of food grain reaches the first origin node as shown in Fig.2, the total units of food grains wasted at this node is equal to $100 \mu_{o 1}$, where $\mu_{o 1}$ is the fraction of food grains wasted at the first origin node. The total amount of food grains that flow out of the node equals $100\left(1-\mu_{o 1}\right)$ units. Based on the routing decision, this quantity is further split and is transported through different destination nodes. Given that $50 \%$ of the outflow from first origin node reaches first

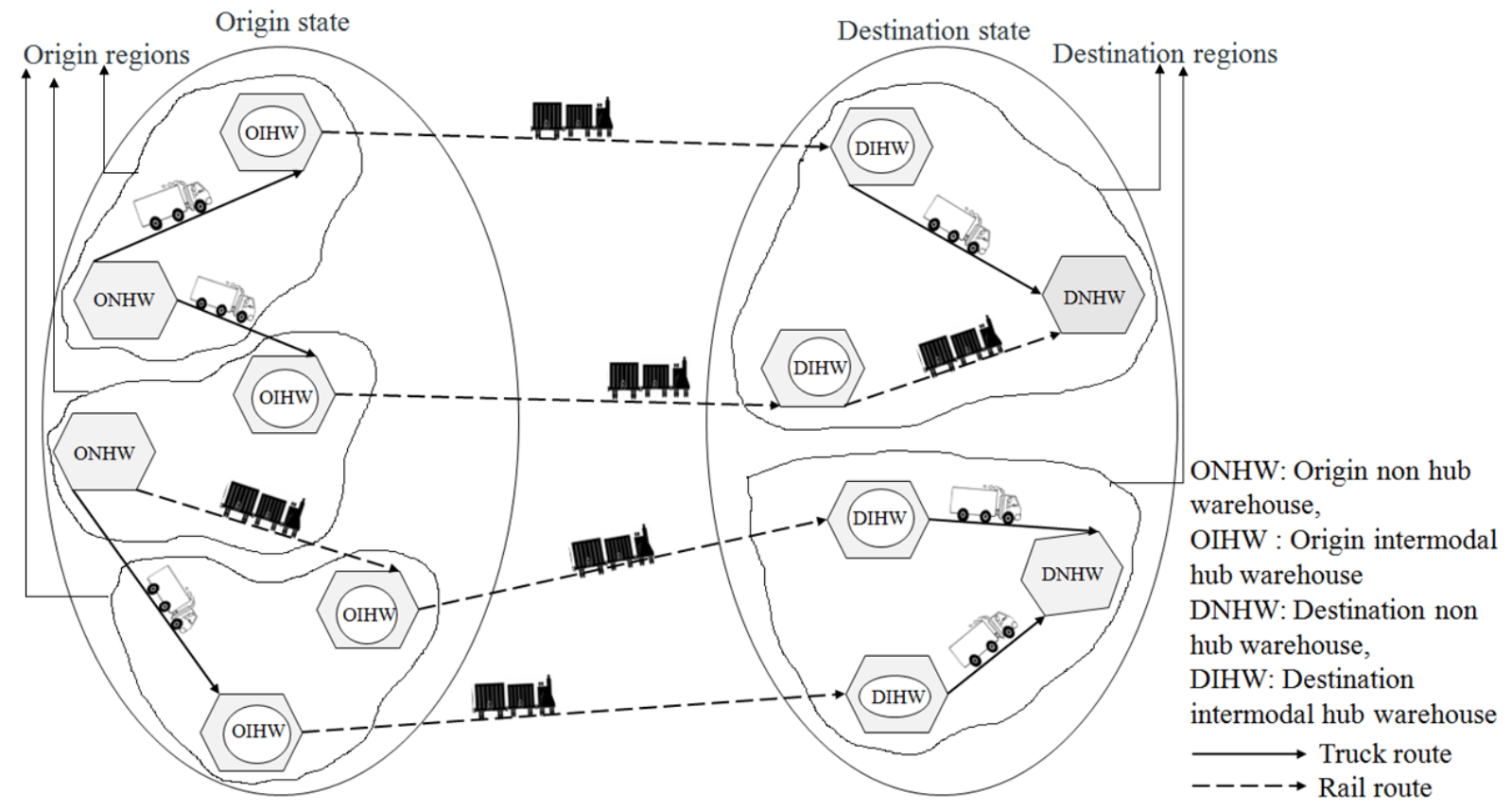

Fig. 1. Overview of food grain supply network

destination node, and similarly the amount of food grains reaching the same node from second and third origin nodes are $10\left(1-\mu_{o 2}\right)$ and $35\left(1-\mu_{o 3}\right)$ units respectively, the total wastages at this hub will amount to $\mu_{d 1}\left[50\left(1-\mu_{o 1}\right)+10\left(1-\mu_{o 2}\right)+35\left(1-\mu_{o 3}\right)\right]$ units, where $\mu_{d 1}$ is the fraction of food grains wasted at the first destination node. The second term represents the total 
amount of food grains reaching the first destination node from all the origin intermodal nodes. The total outgoing quantity of food grains from first destination node after accounting for wastages at origin and destination nodes is given by $\left(1-\mu_{d 1}\right)\left[50\left(1-\mu_{o 1}\right)+10\left(1-\mu_{o 2}\right)+35\left(1-\mu_{o 3}\right)\right]$ as shown in Fig. 2. The following section delineates the mathematical model with its underlying assumptions and detailed description of objective function and constraints.

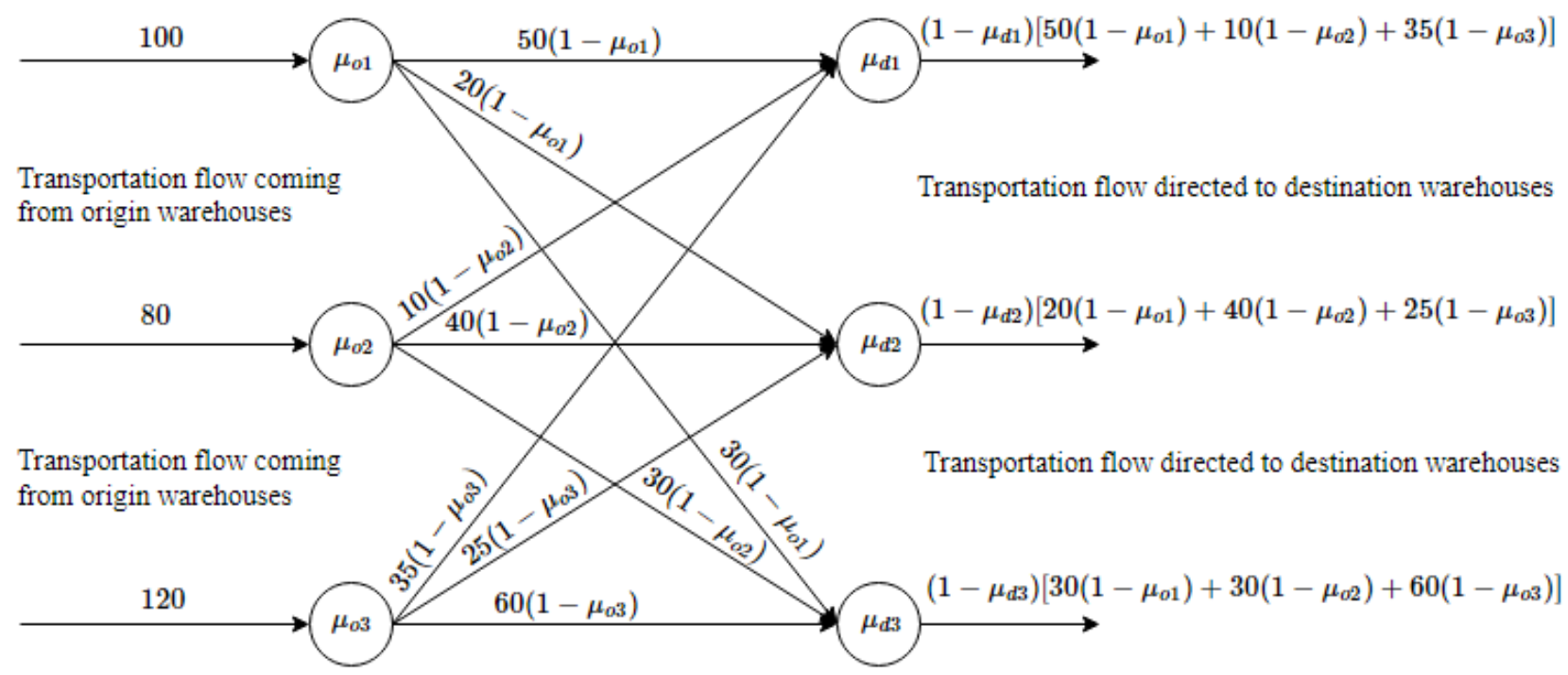

Fig. 2. Intermodal hub transfer linkages considering wastages

\section{Mathematical model}

The proposed model replicates the transportation activities of Indian food grain context where the demand and supply are known prior to start of planning process. Thus, the instantaneous variation in problem parameters is not practically relevant to the problem under study to the best of our knowledge. Previously, authors have dealt with food grain supply chains under deterministic scenario (Mogale et al. (2017), Asgari et al. (2013)). The reason for such treatment is mainly attributed to the following important aspects. The food grain demand is almost constant and is extrapolated from population census data, whereas the abundance in the food grain yield nearly classifies the supply to be of deterministic nature. On the other hand, food grain distribution from abundantly available supply to deterministic demand points with 
efficient utilization of various resources is a striking challenge in Indian context owing to geographically widespread yields. Thus we believe, enforcing probability of different scenarios into the current model would hamper the stated purpose and would reduce the computational efficiency of the proposed approach. Thus the problem aims to streamline sustainable transportation operations under disruption with effective utilization of facility and vehicle resources with deterministic parameters and variables. Hence, the green supply network problem is formulated with following important assumptions:

- All parameters and variables are deterministic in nature

- Homogenous fleet of vehicles is used

- Time consumed for loading/unloading operation is relatively small in comparison with total travel time.

- Food grain wastage due to transportation is considered negligible

- The distance between truck unloading station and train loading station at the intermodal hub is negligible

The symbolic notations consisting of various indices and sets followed for defining various parameters and decision variables of the mathematical formulation are described as follows:

The food grain transportation model is formulated as a constrained multi-objective optimization problem with two objectives $f_{1}$ and $f_{2}$ representing the total supply network cost and total GHG ( $\mathrm{CO}_{2}$ equivalents) emissions respectively. The functions, $f_{1}$ and $f_{2}$ are minimized simultaneously in order to obtain the optimal set of values for warehouse wise shipment quantities, modal choice, vehicle speed and facility allocations. The total supply network cost, $f_{1}$ is expressed as the summation of total transportation cost, total hub location cost, total handling cost at facilities, and total penalty cost of violating service time restrictions. 
The total transportation cost is calculated by splitting the length of the journey in to three sections and subsequently aggregating the cost incurred for each section of the journey. The transportation cost for the initial section is the cost incurred to transport the food grain material from the origin warehouses to the origin hubs either by rail or by road and is represented by Eq. (1) . The transportation cost for the intermediate section is the cost incurred for inter-hub transportation of food grains by rail from origin state hubs to the destination state hubs, mathematically represented by Eq. (2), where, $\mu_{o k}$ is fraction of food grain quantity lost while handling at origin hub $k$, where, $k \in H_{o}$. Finally, transportation cost for the last section of journey is the cost incurred for shipping the food grain quantity from the destination hubs to the warehouses in the destination state. It is represented by Eq. (3), where $\mu_{d m}$ is fraction of food grain quantity lost while handling at destination hub $m$, where, $m \in H_{d}$. The aggregated total transportation cost is obtained by the summation of Eqs. (1), (2), and (3).

$$
\begin{aligned}
& \sum_{t, k, i, p}\left(C_{i p k}^{o \phi} a_{i p k}^{o \phi} \delta_{i p k t}^{o \phi}+C_{i p k}^{o \psi} a_{i p k}^{o \psi} \delta_{i p k t}^{o \psi}\right) \sum_{m, j, q} x_{i p k m t}^{j q} \\
& \sum_{t, m, k} \alpha\left(1-\mu_{o k}\right) C_{k m}^{o d} a_{k m}^{o d} \sum_{i, p, j, q} x_{i p k m t}^{j q} \\
& \sum_{t, m}^{j q}\left(1-\mu_{d m}\right) \sum_{k}\left(1-\mu_{o k}\right) \sum_{i, p, j, q}\left(C_{m j q}^{d \phi} a_{m j q}^{d \phi} \delta_{m j q t}^{d \phi}+C_{m j q}^{d \psi} a_{m j q}^{d \psi} \delta_{m j q t}^{d \phi}\right) x_{i p k m t}^{j q}
\end{aligned}
$$

Eq. (4) determines the total cost of locating intermodal hub facilities at both the origin and destination states. The unit hub location cost for a single facility in this paper is calculated as a sum of cost incurred for land acquisition, construction of storage facilty and infrastructure (railway sidings, weighing bridge and electrification). This is derived based on the availability of data for conducting this study (https://dfpd.gov.in/ppp-storage-scheme.htm). The cost of operations is included in the broader spectrum of food grain handling costs incurred by a single facility which accounts for labour wages, maintaining the equipment and imminent repairs, 
electricity consumption, and quality control involved for handling the food grains as a linear function of quantity handled at the intermodal hub facility. Thus, the total handling cost for the whole shipment is expressed as shown in Eq. (5).

$$
\begin{aligned}
& \sum_{k, t} A_{k} z_{k t}+\sum_{m, t} A_{m} w_{m t} \\
& C_{h}\left[\sum_{t, k, i, p, m, j, q} x_{i p k m t}^{j q}+\sum_{t, m, k}\left(1-\mu_{o k}\right) \sum_{i, p, j, q} x_{i p k m t}^{j q}\right]
\end{aligned}
$$

The time required to reach the destination warehouse $j$ of region $q$ for a food grain consignment starting from origin warehouse $i$ of region $p$ routed through origin hub $k$ and destination hub $m$ in time period $t$ is given by,

$$
\tau_{i p k m t}^{j q}=\frac{a_{i p k}^{o \phi} \delta_{i p k t}^{o \phi}}{v_{\phi}}+\frac{a_{i p k}^{o \psi} \delta_{i p k t}^{o \psi}}{v_{\psi}}+\frac{\beta a_{k m}^{o d} \delta_{k m t}^{o d}}{v_{\psi}}+\frac{a_{m j q}^{d \phi} \delta_{m j q t}^{d \phi}}{v_{\phi}}+\frac{a_{m j q}^{d \psi} \delta_{m j q t}^{d \psi}}{v_{\psi}}, \forall i, \forall p, \forall k, \forall m, \forall j, \forall q, \forall t
$$

where, $\delta_{k m t}^{o d}$ indicates if there is a positive shipment between origin hub $k\left(k \in H_{o}\right)$ and destination hub $m \mid m \in H_{d}$ in time period $t \mid t \in T . \delta_{k m t}^{o d}$ is directly dependent on $\sum_{i, p, j, q} y_{i p k m t}^{j q}$ and is equal to $\left[\sum_{i, p, j, q} y_{i p k m t}^{j q} /\left(1+\sum_{i, p, j, q} y_{i p k m t}^{j q}\right)\right], \forall k, \forall m$ and $\forall t$. Given that, $T_{j q t}$ is the service time upper limit for the food grain demand at warehouse $j \mid j \in W_{q}$ of region $q \mid q \in R_{d}$, the total supply network cost, $f_{1}$ for the first objective is formulated as,

$$
\begin{aligned}
& \text { Minimize } f_{1}=\sum_{t, k, i, p}\left(C_{i p k}^{o \phi} a_{i p k}^{o \phi} \delta_{i p k t}^{o \phi}+C_{i p k}^{o \psi} a_{i p k}^{o \psi} \delta_{i p k t}^{o \psi}\right) \sum_{m, j, q} x_{i p k m t}^{j q}+\sum_{t, m, k} \alpha\left(1-\mu_{o k}\right) C_{k m}^{o d} a_{k m}^{o d} \sum_{i, p, j, q} x_{i p k m t}^{j q} \\
& +\sum_{t, m}\left(1-\mu_{d m}\right) \sum_{k}\left(1-\mu_{o k}\right) \sum_{i, p, j, q}\left(C_{m j q}^{d \phi} a_{m j q}^{d \phi} \delta_{m j q t}^{d \phi}+C_{m j q}^{d \psi} a_{m j q}^{d \psi} \delta_{m j q t}^{d \psi}\right) x_{i p k m t}^{j q}+\sum_{k, t} A_{k} z_{k t}+\sum_{m, t} A_{m} w_{m t} \\
& +\sum_{t, k, i, p, m, j, q} C_{h} x_{i p k m t}^{j q}+\sum_{t, m, k} C_{h}\left(1-\mu_{o k}\right) \sum_{i, p, j, q} x_{i p k m t}^{j q}+\sum_{t, i, p, k, m, j, q} C_{p}\left\{\tau_{i p k m t}^{j q}-T_{j q t}\right\}^{+}
\end{aligned}
$$

where, $\left\{\tau_{i p k m t}^{j q}-T_{j q t}\right\}^{+}=\max \left(0, \tau_{i p k m t}^{j q}-T_{j q t}\right), \forall i, \forall p, \forall k, \forall m, \forall j, \forall q, \forall t$. The second objective minimizes the total GHG emissions, $f_{2}$. In the context of this paper, transportation by vehicles 
and food grain wastages due to improper handling at facilities are primarily held responsible for causing emissions. For simplicity, the fleet of trucks are assumed to be fueled by single fuel (diesel) and the fleet of trains are hauled by electric locomotives. Estimating the GHG emissions (equivalent grams of $\mathrm{CO}_{2}$ released) from vehicles is carried out in three steps. In the first step, calculation of amount of fuel consumed by trucks and conversion of the fuel consumed to equivalent grams of $\mathrm{CO}_{2}$ emission for transportation in the origin and destination states is carried out for trucks. In the second step, electric fuel consumed by trains while conducting transportation in the origin state, destination state and in between the origin and destination states is evaluated. Subsequently in this step, the conversion of fuel consumed to associated $\mathrm{CO}_{2}$ emissions is carried out. The last step calculates the total transportation emissions by all the trucks and trains selected for realizing the food grain demand by aggregating individual emissions for the whole journey.

The liters of fuel consumed by trucks to travel from warehouse $i$ of region $p$ to hub $k, F_{o \phi}$ in the origin state is obtained by rewriting Eq. (B.1) as Eq. (8). Similarly, liters of fuel consumed by trucks to travel from hub $m$ to warehouse $i$ of region $p, F_{d \phi}$ in the destination state is obtained by rewriting Eq. (B.1) as Eq. (9). The amount of fuel consumed is converted to equivalent grams of $\mathrm{CO}_{2}$ emissions using Eqs. (10) and (11) respectively for origin and destination states, where $e_{\phi}$ is the truck emission conversion factor expressed in $\mathrm{gCO}_{2} / \mathrm{l}$.

$$
\begin{gathered}
F_{o \phi}=\sum_{t, k, i, p}\left(\begin{array}{c}
\frac{\mu B V \lambda a_{i p k}^{o \phi} \delta_{i p k t}^{o \phi} l_{i p k t}}{v_{\phi}}+w \gamma \lambda \omega a_{i p k}^{o \phi} \delta_{i p k t}^{o \phi} l_{i p k t} \\
+\gamma \lambda \omega a_{i p k}^{o \phi} \delta_{i p k t}^{o \phi} \sum_{m, j, q} x_{i p k m t}^{j q}+\zeta \gamma \lambda a_{i p k}^{o \phi} \delta_{i p k t}^{o \phi} l_{i p k t} v_{\phi}^{2}
\end{array}\right), \\
\text { where } l_{i p k t}=\left[\frac{\sum_{m, j, q} x_{i p k m t}^{j q}}{C_{\phi} \delta_{i p k t}^{o \phi}+C_{\psi} \delta_{i p k t}^{o \psi}}\right\rceil, \forall i, \forall p, \forall k, \forall t
\end{gathered}
$$


$F_{d \phi}=\sum_{t, m, j, q}\left(\begin{array}{l}\frac{\mu B V \lambda a_{m j q}^{d \phi} \delta_{m j q t}^{d \phi} h_{m j q t}}{v_{\phi}}+w \gamma \lambda \omega a_{m j q}^{d \phi} \delta_{m j q t}^{d \phi} h_{m j q t}+\gamma \lambda \omega a_{m j q}^{d \phi} \delta_{m j q t}^{d \phi}\left(1-\mu_{d m}\right) \sum_{k}\left(1-\mu_{o k}\right) \sum_{i, p} x_{i p k m t}^{j q} \\ +\zeta \gamma \lambda a_{m j q}^{d \phi} h_{m j q t} v_{\phi}^{2}\end{array}\right)$

where $h_{m j q t}=\left\lceil\frac{\left(1-\mu_{d m}\right) \sum_{k}\left(1-\mu_{o k}\right) \sum_{i, p} x_{i p k m t}^{j q}}{C_{\phi} \delta_{m j q t}^{d \phi}+C_{\psi} \delta_{m j q t}^{d \psi}}\right], \forall m, \forall j, \forall q, \forall t$

$\mathrm{X}_{o \phi}=e_{\phi} F_{o \phi}$

$\mathrm{X}_{d \phi}=e_{\phi} F_{d \phi}$

The total electric fuel consumed during rail transport for the current problem in the first, second and third sections of the journey is derived using Eq. (C.1) and Table C.1 as shown in Eqs. (12), (13), and (14) respectively. The coefficients of fuel consumption equation for the case of multiple wagons and multiple trains are estimated by extending the single wagon case. The dependent variables that capture number of trains or trucks passing through a given arc in the first and third sections, $l_{i p k t}$ and $h_{\text {mjqt }}$ are defined earlier in Eqs. (8) and (9) respectively. The number of trains passing through an arc in the second section is equal to $\left\lceil\left(1-\mu_{o k}\right) \sum_{i, p, j, q} x_{i p k m t}^{j q} / C_{\psi}\right\rceil$ and is denoted by $n_{k m t}$. The weight of shipment, $W_{s h i p}$ is obtained by converting the total quantity of food grains transported through the arc to equivalent weight (in $\mathrm{N})$. The quantity shipped in the first section of the journey from origin warehouse $i$ of region $p$ to origin hub $k$ is equal to $\sum_{m, j, q} x_{i p k m t}^{j q}$. The food grain shipment quantities from origin hub $k$ to destination hub $m$ in the second section, after subtracting the wastages at origin hubs is equal to $\left(1-\mu_{o k}\right) \sum_{i, p, j, q} x_{i p k m t}^{j q}$. Similarly, the shipment quantities corresponding to the third section from destination hub $m$ to destination warehouse $j$ of region $q$ after considering wastages at origin and destination hubs is equal to $\left(1-\mu_{d m}\right) \sum_{k}\left(1-\mu_{o k}\right) \sum_{i, p} x_{i p k m t}^{j q}$. The fuel 
consumed by trains is converted to equivalent grams of $\mathrm{CO}_{2}$ by following Eqs. (15), (16), and

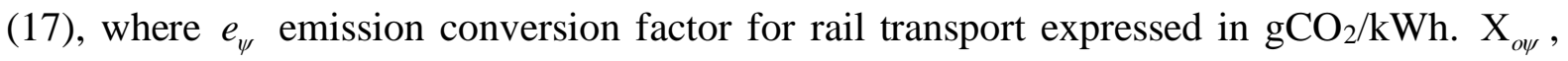
$\mathrm{X}_{o d}$, and $\mathrm{X}_{d \psi}$ are the equivalent $\mathrm{CO}_{2}$ emissions for the first, second and third sections of the journey respectively. Finally, the actual transportation emissions from trucks and trains for the whole journey, $\mathrm{X}_{\text {tran }}$ is the summation of individual emission components as shown in Eq. (18).

$$
\begin{aligned}
& F_{o \psi}=R \sum_{t, k, i, p}\left(u_{i p k t}^{o r}+u_{i p k t}^{o s} v_{\psi}+u_{i p k t}^{o c} v_{\psi}{ }^{2}\right) a_{i p k}^{o \psi} \delta_{i p k t}^{o \psi} \\
& F_{o d}=R \sum_{t, k, m}\left(u_{k m t}^{o d}+u_{k m t}^{o d} v_{\psi}+u_{k m t}^{o d} v_{\psi}{ }^{2}\right) a_{k m}^{o d} \delta_{k m t}^{o d} \\
& F_{d \psi}=R \sum_{t, m, j, q}\left(u_{m j q t}^{d}+u_{m j q t}^{d} v_{\psi}+u_{m j q t}^{d} v_{\psi}{ }^{2}\right) a_{m j q}^{d \psi} \delta_{m j q t}^{d \psi} \\
& \mathrm{X}_{o \psi}=e_{\psi} F_{o \psi} \\
& \mathrm{X}_{o d}=e_{\psi} F_{o d} \\
& \mathrm{X}_{d \psi}=e_{\psi} F_{d \psi} \\
& \mathrm{X}_{t r a n}=\mathrm{X}_{o \phi}+\mathrm{X}_{o \psi}+\mathrm{X}_{o d}+\mathrm{X}_{d \phi}+\mathrm{X}_{d \psi}
\end{aligned}
$$

Emissions due to wastages at facilities is estimated using Eq. (19), where, $e$ is the $\mathrm{CO}_{2}$ emissions conversion factor for unit quantity of food grains wasted at intermodal hub facilities and $\mathrm{X}_{f a c}$ is the total equivalent grams of $\mathrm{CO}_{2}$ emissions due to wastages at both origin and destination hubs.

$$
\mathrm{X}_{f a c}=e\left[\sum_{t, k} \mu_{o k} \sum_{i, p, m, j, q} x_{i p k m t}^{j q}+\sum_{t, m} \mu_{d m} \sum_{k}\left(1-\mu_{o k}\right) \sum_{i, p, j, q} x_{i p k m t}^{j q}\right]
$$

Combining Eqs. (18) and (19), the second objective is formulated as,

Minimize $f_{2}=\mathrm{X}_{\text {tran }}+\mathrm{X}_{f a c}$ 
The constraint set for this problem is described in Eqs. (21)-(48). Eq. (21) ensures that there is positive quantity of food grains flowing through a route if and only if there exists a route in that direction. The least integer function defined in Eq. (22) avoids empty vehicle transport between the origin and destination warehouses. Eqs. (23) and (24) state that the route link between the decision variables that determine interstate food grain shipment quantity and location of intermodal hub facilities. Eq. (25) and (26) enforce constraints that only one mode of transport is allowed to be selected for a route which exists between a warehouse and a hub. However, through these constraints the model accommodates for the possibility to choose between rail or road provided there exists a full route between origin and destination warehouses. The restriction on maximum number of hubs that can be allocated in any time period is imposed by Eqs. (27) and (28). Eq. (29) ensures that demand of food grains at the destination warehouses is satisfied after considering the wastage occurred at intermodal hubs. Eq. (30) represents the set of constraints which ensure that the food grain quantity dispatched to all the warehouses in a particular time period is less than the available inventory in that period. Eq. (31) represents flow balance constraints.

$$
\begin{aligned}
& x_{i p k m t}^{j q} \leq M y_{i p k m t}^{j q}, \forall i, \forall p, \forall k, \forall m, \forall j, \forall q, \forall t \\
& y_{i p k m t}^{j q}=\left[\frac{x_{i p k m t}^{j q}}{1+x_{i p k m t}^{j q}}\right], \forall i, \forall p, \forall k, \forall m, \forall j, \forall q, \forall t \\
& \sum_{i, p, m, j, q} y_{i p k m t}^{j q} \leq\left(\left|\bigcup_{p=1}^{\left|R_{o}\right|} W_{p}\right|\left|H_{d}\right|\left|\bigcup_{q=1}^{\left|R_{o}\right|} W_{p}\right|\right) z_{k t}, \forall k, \forall t \\
& \sum_{i, p, k, j, q} y_{i p k m t}^{j q} \leq\left(\left|\bigcup_{p=1}^{\left|R_{o}\right|} W_{p}\right|\left|H_{o}\right|\left|\bigcup_{q=1}^{\left|R_{d}\right|} W_{q}\right|\right) w_{m t}, \forall m, \forall t \\
& y_{i p k m t}^{j q} \leq \delta_{i p k t}^{o \phi}+\delta_{i p k t}^{o \psi}, \forall i, \forall p, \forall k, \forall m, \forall j, \forall q, \forall t \\
& y_{i p k m t}^{j q} \leq \delta_{m j q t}^{d \phi}+\delta_{m j q t}^{d \psi}, \forall i, \forall p, \forall k, \forall m, \forall j, \forall q, \forall t
\end{aligned}
$$




$$
\begin{aligned}
& \sum_{k} z_{k t}=b_{o}, \forall t \\
& \sum_{m} w_{m t}=b_{d}, \forall t \\
& \sum_{m}\left(1-\mu_{d m}\right) \sum_{k}\left(1-\mu_{o k}\right) \sum_{i, p} x_{i p k m t}^{j q} \geq D_{j t}^{q}, \forall j, \forall q, \forall t \\
& \sum_{k, m, j, q} x_{i p k m t}^{j q} \leq I_{i p t}, \forall i, \forall p, \forall t \\
& I_{i p t}=I_{i p(t-1)}+P_{i p t}-\sum_{k, m, j, q} x_{i p k m t}^{j q}, \forall i, \forall p, \forall t
\end{aligned}
$$

Eqs. (32) and (33) enforce maximum handling capacity restriction at origin and destination hubs respectively. Eq. (34) states that the total wastages occurred at intermodal hubs should not be crossing a fixed upper limit. It is important to adhere to vehicle capacity restrictions. Thus. Eqs. (35), (36) and (37) are written to take care of this concern for origin warehouses, origin hubs and destination hubs respectively. The binary parameter, $\varepsilon_{i p}$ (defined in Appendix A) is incorporated in Eq. (35) to establish the difference between hub nodes and non-hub nodes. $\pi_{i p t}^{o \phi}$ and $\pi_{m t}^{d \phi}$ are binary variables which are equal to one if there exists at least one truck route that starts from origin non- hub warehouse, $i$ of region $p$ and destination hub $m$ respectively. Similarly, $\pi_{i p t}^{o \psi}$ and $\pi_{m t}^{d \psi}$ are binary variables which are equal to one if there exists at least one train route that starts from a origin non- hub warehouse, $i$ of region $p$ and destination hub $m$ respectively. Eqs. (38) and (39) ensure that there is no transport in between the hubs in the origin and destination state respectively. Finally, Eqs. (40)-(48) indicate non-negativity and integrality constraints for all continuous and binary variables.

$$
\begin{aligned}
& \sum_{i, p, m, j, q} x_{i p k m t}^{j q} \leq U_{k} z_{k k t}, \forall k, \forall t \\
& \sum_{k}\left(1-\mu_{o k}\right) \sum_{i, p, j, q} x_{i p k m t}^{j q} \leq U_{m} w_{m t}, \forall m, \forall t \\
& \sum_{k} \mu_{o k} \sum_{i, p, m, j, q} x_{i p k m t}^{j q}+\sum_{m} \mu_{d m} \sum_{k}\left(1-\mu_{o k}\right) \sum_{i, p, j, q} x_{i p k m t}^{j q} \leq K, \forall t
\end{aligned}
$$




$$
\begin{aligned}
& \left(1-\varepsilon_{i p}\right) \sum_{k, m, j, q} x_{i p k m t}^{j q} \leq \rho_{i p t}^{o \phi} \pi_{i p t}^{o \phi} C_{\phi}+\rho_{i p t}^{o \psi} \pi_{i p t}^{o \psi} C_{\psi}, \\
& \text { where, } \pi_{i p t}^{o \phi}=\left\lceil\frac{\sum_{k} \delta_{i p k t}^{o \phi}}{1+\sum_{k} \delta_{i p k t}^{o \phi}}\right\rceil \text { and } \pi_{i p t}^{o \psi}=\left\lceil\frac{\sum_{k} \delta_{i p k t}^{o \psi}}{1+\sum_{k} \delta_{i p k t}^{o \psi}}\right\rceil, \forall i, \forall p, \forall t \\
& \left(1-\mu_{o k}\right) \sum_{i, p, m, j, q} x_{i p k m t}^{j q} \leq \sigma_{k t} z_{k t} C_{\psi}, \forall k, \forall t \\
& \left(1-\mu_{d m}\right) \sum_{k}\left(1-\mu_{o k}\right) \sum_{i, p, j, q} x_{i p k m t}^{j q} \leq \rho_{m t}^{d \phi} \pi_{m t}^{d \phi} C_{\phi}+\rho_{m t}^{d \psi} \pi_{m t}^{d \psi} C_{\psi}, \\
& \text { where, } \pi_{m t}^{d \phi}=\left\lceil\frac{\sum_{j, q} \delta_{m j q t}^{d \phi}}{1+\sum_{j, q} \delta_{m j q t}^{d \phi}}\right\rceil \text { and } \pi_{m t}^{d \psi}=\left\lceil\frac{\sum_{j, q} \delta_{m j q t}^{d \psi}}{1+\sum_{j, q} \delta_{m j q t}^{d \psi}}\right\rceil, \forall m, \forall t \\
& x_{i p k m t}^{j q}=0, \forall i, \forall p, \forall j, \forall q, \forall t,((i, p), k) \in H_{o} \\
& x_{i p k m t}^{j q}=0, \forall i, \forall p, \forall j, \forall q, \forall t,((j, q), m) \in H_{d} \\
& x_{i p k m t}^{j q} \geq 0, \forall i, \forall p, \forall j, \forall q, \forall k, \forall m, \forall t \\
& v_{\phi}, v_{\psi} \geq 0 \\
& y_{i p k m t}^{j q} \in\{0,1\}, \forall i, \forall p, \forall j, \forall q, \forall k, \forall m, \forall t \\
& z_{k t} \in\{0,1\}, \forall k, \forall t \\
& w_{m t} \in\{0,1\}, \forall m, \forall t \\
& \delta_{i p k t}^{o \phi}, \delta_{i p k t}^{o \psi} \in\{0,1\}, \forall i, \forall p, \forall k, \forall t \\
& \delta_{m j q t}^{d \phi}, \delta_{m j q t}^{d \psi} \in\{0,1\}, \forall m, \forall j, \forall q, \forall t \\
& \pi_{i p t}^{o \phi}, \pi_{i p t}^{o \psi} \in\{0,1\}, \forall i, \forall p, \forall t \\
& \pi_{j q t}^{d \phi}, \pi_{j q t}^{d \psi} \in\{0,1\}, \forall j, \forall q, \forall t
\end{aligned}
$$

The following unique aspects of the proposed formulation sets the problem apart from the previous studies in green supply chain literature. Firstly, the quantitative modelling of GHG emissions in food grain supply chain context with simultaneous focus to minimize the total supply network costs and total cost GHG emissions in the presence of wastages is newly incorporated in this study. Secondly, the idea of reducing food grain wastages is captured in 
this model by inclusion of an explicit constraint in Eq. (34). Further, demand constraints, hub capacity restrictions, flow balance equations, vehicle capacity restrictions, and multi-modal considerations are specifically modelled to address the practical food grain transportation problem.

\section{Solution methodology}

The solution for a nonlinear multi-objective optimization problem is found to be increasingly computationally intractable for growingly larger instances. The green supply network optimization formulated in this work is a generalization of two different class of problems which are NP-hard, pollution routing problem (Kumar et al. 2016) and p-hub median problem (Ishfaq and Sox, 2011). Further, it includes additional complexities with respect to multi-modal allocations and food grain wastages. Therefore, by inference the proposed problem is NP hard and cannot be solved in polynomial time using the existing set of exact optimization approaches. Given the highly complex nature of the proposed formulation, probability of achieving higher computational times and inaccurate solutions on further decomposition and linearization of the objective function and constraints of the aforementioned problem is considerably high. These shortcomings and inability of exact solution approaches to deal with large problems in real time narrow down the scope of their applicability to solve mixed integer non-linear multi-objective problems. Su and Chi, (2017) proposed and demonstrated the superiority of multi-objective particle swarm optimization with differential evolution (MOPSODE) over other metaheuristics for solving nonlinear large scale multi-objective problems. The governing principles of PSODE and MOPSODE are provided in Appendices D and E respectively. Section 5.1 delineates detail description of modified MOPSODE algorithm employed in this paper. 


\subsection{MOPSODE with new constraint violation based penalty allocation}

The modified MOPSODE version proposed in this paper incorporates a new constraint violation based search guidance scheme to enhance the random search process. The global fitness, $G_{i}(\mathrm{X})$ of $i^{\text {th }}$ objective for a given solution vector $\mathrm{X}$ is evaluated according to Eq. (49). Here, $V_{n}(\mathrm{X})$ is the degree of violation of $n^{\text {th }}$ constraint, $\pi_{n}$ is penalty for violating the constraint, and $Y_{i}(\mathrm{X})$ is the value of $i^{\text {th }}$ objective function.

$$
G_{i}(\mathrm{X})=Y_{i}(\mathrm{X})+\sum_{n} \pi_{n} V_{n}(\mathrm{X}), \forall i
$$

Given that $H_{l}(\mathrm{X}) \leq B_{l}$ and $H_{m}(\mathrm{X})=B_{m}$ are the set of inequality and equality constraints of the problem respectively, $V_{n}(\mathrm{X})$ is calculated differently for each of these cases as shown in Eq. (50)

$$
V_{n}(\mathrm{X})= \begin{cases}\left\{H_{l}(\mathrm{X})-B_{l}\right\}^{+} & \text {if } n=l \\ \left|H_{m}(\mathrm{X})-B_{m}\right| & \text { if } n=m\end{cases}
$$

The basic MOPSODE version handles violated constraints according to mid-point reflection rule (Eq. (D.6)). Attributed to high difference in violated and corrected offsprings, mid-point reflection performs significantly well in the initial stages of execution, however fails to accurately converge in the penultimate phases of the search process. Selecting a finer constraint handling strategy in the final stages would facilitate accurate learning of near optimal pareto fronts. In this respect, for every unsatisfied constraint $l$ in iteration $t$, a refined constraint handling technique as shown in Eq. (51) is used in this work. For each violated constraint $l$, $w_{l}^{t}$ in Eq. (51) represents the maximal element of the set that contains all continuous decision variables having positive correlation with degree of violation, where, $k$ is used to represent any element of the set and $N_{l}$ is the total number of elements in that set. 


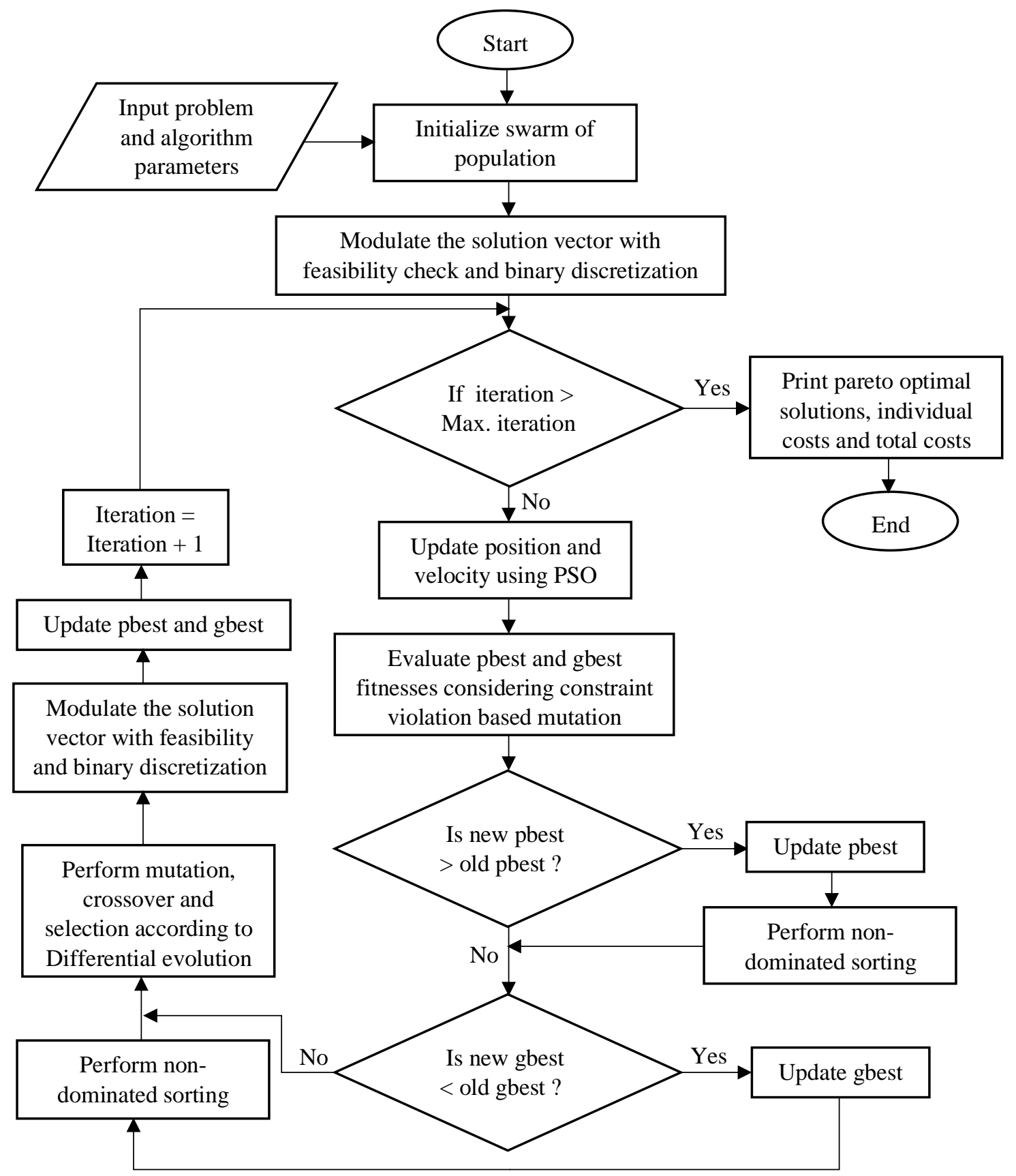

Fig. 3. Flow diagram for MOPSODE

The flow diagram in Fig. 3 presents the step wise implementation of modified MOPSODE and Fig. 4 narrates its pseudocode. Fig. 5 illustrates the solution encoding of a single particle chosen from the population swarm for medium size problem (described in Appendix E).

According to Eq. (51), the value of maximal element $\left(w_{l}^{t}\right)$ is reduced by $p \%$ for the next iteration. Setting too high value for $p$ would result to excessive scattering of solutions, 
whereas on the contrary, a lower value would reap insignificant benefits. Thus, it is found that $p$ should be chosen appropriately in the range [15 20].

$$
w_{l}^{t}=\left(1-\frac{p}{100}\right) w_{l}^{t}, \forall k, \forall l, \quad \text { where } w_{l}^{t}=\max \left[\bigcup_{k=1}^{N_{l}}\left\{w_{k l}^{t}\right\}\right]
$$

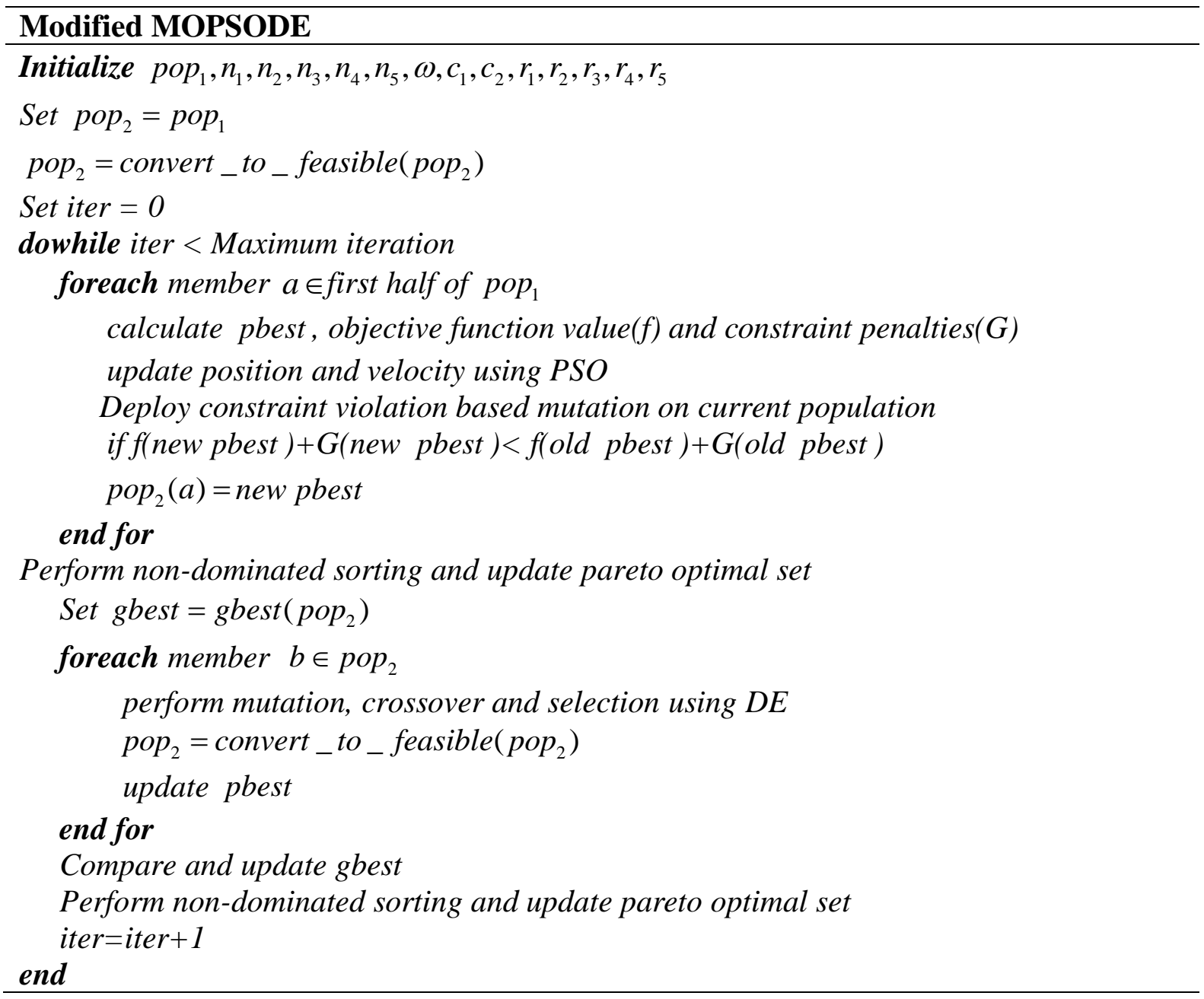

Fig. 4. Pseudocode for modified MOPSODE

The advantages of the proposed approach are enlisted as follows. First, it requires lesser number of iterations to arrive at quality solutions as the particle best rapidly betters itself owing guided by efficient constraint violation and feasible boundary restriction schemes. Second, the hybrid approach escapes local entrapment by virtue of embedded mutation strategies and constraint handling schemes which help to maintain adequate solution diversity and accuracy in the random search process. 

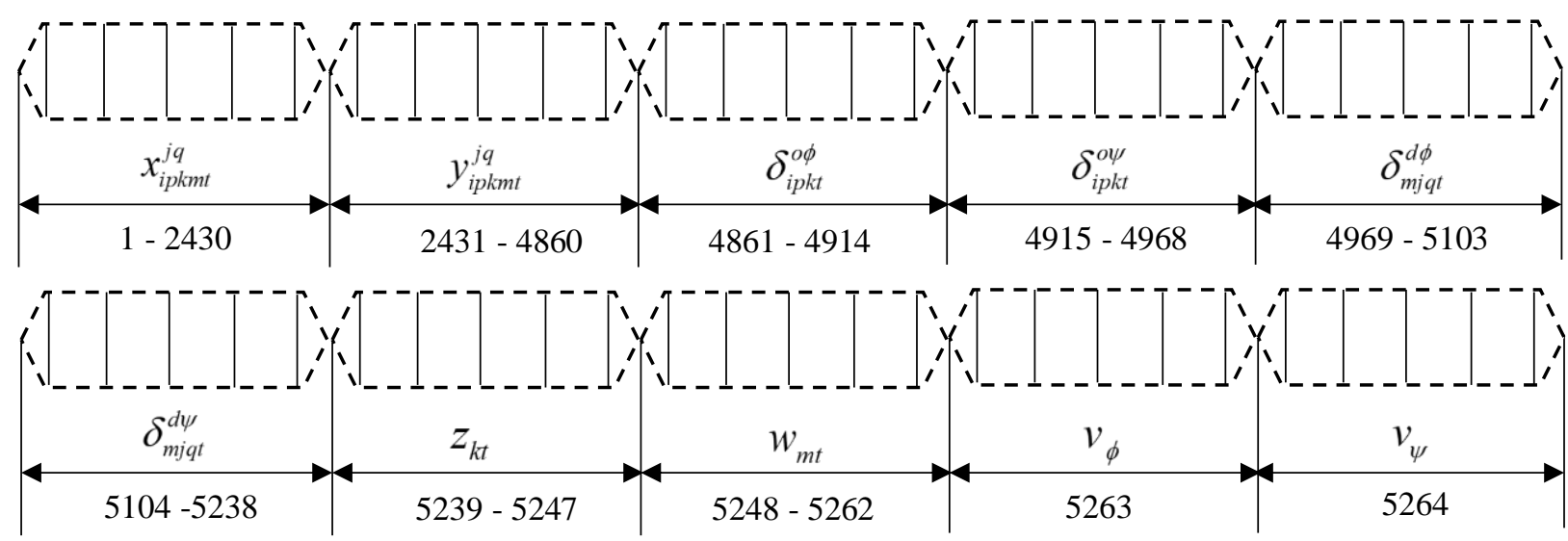

Fig. 5. Solution encoding of single particle for medium size data set.

\subsection{Time complexity}

The time complexity of particle swarm optimization as well as differential evolution algorithms are of the order $O(N D)$, where $N$ is the population size and $D$ is the dimension. Therefore the combined version also solves the problem within $O(N D)$. However, in the multi-objective version the complexity in time is dominated by the calculation of crowding distance and nondominated sorting operations. The crowding distance is calculated for each member of the archive that stores the set of non-dominated individuals of the current iteration. For a problem with $M$ objectives, the complexity at which it is computed is a non-linear function of total number of members in the archive set, $A$ which is given by $O(M A \log A)$. The time consumed by non-dominated sorting operation is of the order $O\left(M N^{2}\right)$, given that the scheme of sorting follows $O\left(N^{2}\right)$ for a single objective problem. The above computational complexities have been calculated for a given size of problem. Increasing the problem size would drastically effect the time complexity. Therefore, it is important to analyze the complexity in time as a function different problem inputs. Given that, for a particular instance of the problem, $n_{1}, n_{2}, n_{3}, n_{4}$, and $n_{5}$ denote the number of origin warehouses, origin hubs, destination hubs, destination warehouses and time periods respectively, the general expressions for aggregated total number of variables $\left(\Omega_{1}\right)$ and constraints $\left(\Omega_{2}\right)$ for the problem instance are shown in Eqs. (52) and 
(53). For each instance, the problem has to deal with $O\left(n_{1} n_{2} n_{3} n_{4} n_{5}\right)$ variables and constraints respectively.

$\Omega_{1}=n_{5}\left(2 n_{1} n_{2} n_{3} n_{4}+2 n_{1} n_{2}+2 n_{3} n_{4}+n_{2}+n_{3}\right)+2$

$\Omega_{2}=n_{5}\left(6 n_{1} n_{2} n_{3} n_{4}+4\left(n_{1}+n_{2}+n_{3}\right)+2 n_{4}+2 n_{3} n_{4}+2 n_{1} n_{2}+3\right)+4$

The composition of number variables and constraints for the problem sets considered are shown in Table 2.

Table 2 Problem set description

\begin{tabular}{llllll}
\hline $\begin{array}{l}\text { Problem } \\
\text { set }\end{array}$ & $\begin{array}{l}\text { Origin } \\
\text { regions }\end{array}$ & $\begin{array}{l}\text { Destination } \\
\text { regions }\end{array}$ & $\begin{array}{l}\text { Configuration } \\
\left(n_{1}, n_{2}, n_{3}, n_{4}, n_{5}\right)\end{array}$ & $\begin{array}{l}\text { Number of } \\
\text { variables }\end{array}$ & $\begin{array}{l}\text { Number of } \\
\text { constraints }\end{array}$ \\
\hline Small & 3 & 3 & $(5,3,3,5,2)$ & 1034 & 2938 \\
Medium & 3 & 4 & $(6,3,5,9,3)$ & 5264 & 15193 \\
Large & 3 & 4 & $(10,4,4,10,3)$ & 10142 & 29569 \\
\hline
\end{tabular}

\section{Experiments}

The data for experimentation is inspired from food grain transportation scenario in Southern India. Geographically, the country is divided into a number of states which are home to millions of people who majorly consume food grains as staple food. Consumption of wheat is dominant in the northern part of the sub-continent whereas rice in the southern part. Owing to the wide geographical spread of territories and significant imbalance between the supply and demand of food grains, an efficient transportation plan with aim to reduce the occurrence of food grain wastages across the Indian food grain supply network is much required. As discussed in the introduction, the need for sustainable design in the aforementioned context is imminent due to the projected rise in the temperature due to global warming and contribution of food grains towards total CO2 emissions (Parikh et al. 2009). The total movement of food grains in India is estimated to reach 45 million metric ton by 2020 (interpolated based on facts revealed by CAG Report, 2013). Considering the large scale of transportation and intermodal transfer operations pertaining to this shipment from food grain surplus to deficit regions, there is huge 
scope for reducing wastages with simultaneous focus to minimize supply network cost and emissions. The proposed model is tested on different instances inspired from the aforementioned real case. For simulation, two geographical territories Tamil Nadu and Andhra Pradesh are chosen as origin and destination states. The demand for rice in Tamil Nadu is satisfied from the surplus stock available at Andhra Pradesh for multiple periods. The model is tested on three samples of data with increasing problem size (increasing number of variables). The data was collected from field visits and various online sources (http:/fci.gov.in, http://pdsportal.nic.in/main.aspx,https://www.fois.indianrail.gov.in/foisweb/view/qry/TQ_Frg tCalcIN.jsp, etc.). The region-wise distribution of warehouses for small, medium and large size instances are shown in Table F.1. Table F.2 describes the numerical values adopted by different parameters of the problem.

Further to this, the paper analyzes the effect of considering different levels of hub location, food grain wastages and intermodal hub capacities on the supply network cost and GHG emission through sensitivity analysis. Tables F.3 and F.4 describe the different level combinations adopted with respect to food grain wastage threshold and intermodal hub capacity in this study. Five different hub location levels are considered, the details of which, are deliberated in the subsequent section. In total, 14 experiments are conducted with varying levels of each one of the aforementioned factors while keeping the other fixed on medium size problem. All the experiments are undertaken with tuned parameter settings as listed in Tables F.5 and F.6. The detail discussion on results and insights gained through further analysis are presented in the next section.

\section{Result and discussion}

MOPSODE was implemented in MATLAB and executed on Windows 8, 64-bit Operating System consisting of $8 \mathrm{~GB}$ RAM and Intel Core i7 $1.8 \mathrm{GHz}$ processor. The experiments are conducted for small medium large size datasets (Table 2) using MOPSODE and NSGA-II. 
Results verify that with slightly higher CPU time MOPSODE, guarantees higher quality of solution than NSGA-II (Table 3). The percentage decrease in total supply network cost and emissions obtained by the proposed approach was observed to be 13.67 and 6.63 for the small, 7.03 and 1.45 for medium and, 8.01 and 10 for the large problems respectively. The computational time for MOPSODE over NSGA-II is found to increase at most by $19 \%$ amongst all the cases which is attributed to the greater number of evolutionary operators in the former approach than the later. The increase in computational time is a good compromise considering the ability of the metaheuristic to solve the complex problem in polynomial time. Figs. 6 (a), (b), and (c) show the highest ranked optimal pareto fronts obtained for all the three instances by MOPSODE and NSGA-II. The graphs indicate that the non-dominated front obtained by using MOPSODE significantly dominates the front obtained by using NSGA-II, thereby, validating the superiority of MOPSODE over NSGA-II for solving small, medium, and large scale problems. Subsequently, the decision variables including shipment quantity, route selection, hub location and vehicle velocities pertaining to the global optimal pareto fronts are evaluated. The emphasis of the discussion carried out in this research is much focused towards simultaneous consideration of cost and environmental concerns. To capture the economic impact of environmental objective, GHG emission is approximated as GHG emission cost by multiplying with a suitable cost conversion factor (carbon tax). The price of carbon tax for the conversion is chosen to lie between Rs 140 per ton of $\mathrm{CO}_{2}$. Subsequently, the average nondominated total cost pertaining to rank 1 solutions for all the instances is computed (Table 3 ) and is found to be less for MOPSODE as compared to NSGA-II by $7.25 \%, 10.77 \%$ and $13.95 \%$ respectively for the different problem sizes.

In most cases, the appropriate choice of the compromising solution to a multi-objective problem depends primarily on the relative priority of individual objectives to the decision makers. The set of non-dominated pareto fronts plotted in Figs. 7 (a), (b) and (c) provides a 
representative subset of solutions for each data set. The range of vehicle velocities were found to lie between $45-65 \mathrm{~km} / \mathrm{hr}$ for trucks and $26-38 \mathrm{~km} / \mathrm{hr}$ for trains in small case, $40-46 \mathrm{~km} / \mathrm{hr}$ for trucks and 21-26 km/hr for trains in medium case, whereas $45-54 \mathrm{~km} / \mathrm{hr}$ for trucks and 24-39 $\mathrm{km} / \mathrm{hr}$ in large case. Accordingly, the best three ranks (rank 1, rank 2, and rank 3) of pareto solutions generated using MOPSODE pertaining to problem sets 1-3 are reported in Table 4. For instance, choosing a point from the bottom right positions of the coordinate axes in Figs. 7 (a), (b), and (c), would certainly ensure low GHG emission costs due to lower levels of vehicle velocity. Having said so, the advantages gained by achieving lower emissions are overshadowed by unaffordably high shipment costs. On the contrary, solution points to the upper left portion of the coordinate axes guarantee low shipment cost but fail to offer economically viable environmental benefits ascribed to higher vehicle velocity levels and corresponding high GHG emissions cost. In alignment with the set objectives, economic and environmental concerns are considered equally important in this paper to harbor holistic advantage. Thus, a compromising solution as highlighted in Figs. 7 (a), (b), and (c) is chosen to ensure environmentally conscious transport of food grain shipments.

Table 3 MOPSODE and NSGA-II results

\begin{tabular}{llllll}
\hline Algorithm & $\begin{array}{l}\text { Problem } \\
\text { set }\end{array}$ & $\begin{array}{l}\text { Supply network } \\
\text { cost (bn Rs) }\end{array}$ & $\begin{array}{l}\text { GHG emissions } \\
(\mathrm{kt} \mathrm{CO})\end{array}$ & $\begin{array}{l}\text { Average non- } \\
\text { dominated overall } \\
\text { cost (bn Rs) }\end{array}$ & $\begin{array}{l}\text { CPU } \\
\text { time (s) }\end{array}$ \\
\hline MOPSODE & Small & 51.7 & 12 & 57.6 & 127 \\
& Medium & $1,365.6$ & 204.7 & $1,387.4$ & 1,335 \\
& Large & $3,555.2$ & 617.9 & $3,765.4$ & 2,347 \\
\hline NSGA-II & Small & 59.9 & 12.9 & 67 & 108 \\
& Medium & $1,468.8$ & 207.6 & $1,554.9$ & 1,137 \\
& Large & $3,864.9$ & 686.4 & 4,060 & 1,967 \\
\hline
\end{tabular}


Table 4 Pareto optimal costs obtained by MOPSODE for all problem sets

\begin{tabular}{|c|c|c|c|c|c|c|c|c|c|}
\hline \multirow{2}{*}{$\begin{array}{l}\text { Pareto } \\
\text { solution }\end{array}$} & \multicolumn{3}{|l|}{ Small } & \multicolumn{3}{|c|}{ Medium } & \multicolumn{3}{|l|}{ Large } \\
\hline & $\begin{array}{l}\text { Front } \\
\text { rank }\end{array}$ & $\begin{array}{l}\text { Supply network } \\
\text { cost (bn Rs) }\end{array}$ & $\begin{array}{l}\text { GHG emission } \\
(\mathrm{kt} \mathrm{CO} 2)\end{array}$ & $\begin{array}{l}\text { Front } \\
\text { rank }\end{array}$ & $\begin{array}{l}\text { Supply network } \\
\text { cost (bn Rs) }\end{array}$ & $\begin{array}{l}\text { GHG emission } \\
(\mathrm{kt} \mathrm{CO} 2)\end{array}$ & $\begin{array}{l}\text { Front } \\
\text { rank }\end{array}$ & $\begin{array}{l}\text { Supply network } \\
\text { cost (bn Rs) }\end{array}$ & $\begin{array}{l}\text { GHG emission } \\
(\mathrm{kt} \mathrm{CO} 2)\end{array}$ \\
\hline 1 & 1 & 44.1 & 13.6 & 1 & $1,263.4$ & 205.2 & 1 & $3,936.3$ & 610.2 \\
\hline 2 & 1 & 80.8 & 11.4 & 1 & $1,243.3$ & 205.7 & 1 & $3,602.9$ & 613.8 \\
\hline 3 & 1 & 51.7 & 12 & 1 & $1,365.6$ & 204.7 & 1 & $3,851.2$ & 612 \\
\hline 4 & 1 & 48.8 & 12.2 & 1 & 1,413 & 201 & 1 & $3,555.2$ & 617.9 \\
\hline 5 & 1 & 54.3 & 11.9 & 2 & 1,508 & 204 & 1 & $3,432.3$ & 767.3 \\
\hline 6 & 2 & 54.1 & 12.9 & 2 & $1,471.5$ & 204.8 & 1 & $3,489.5$ & 703.4 \\
\hline 7 & 2 & 53.2 & 13.6 & 2 & $1,279.7$ & 208.6 & 2 & $3,581.4$ & 619.1 \\
\hline 8 & 2 & 47.5 & 14.5 & 2 & $1,305.5$ & 208.2 & 2 & $4,358.8$ & 611.7 \\
\hline 9 & 2 & 60.3 & 12 & 2 & $1,409.4$ & 205.3 & 2 & $4,017.9$ & 612.2 \\
\hline 10 & 2 & 55.9 & 12.5 & 2 & 1,343 & 206 & 2 & $3,966.6$ & 614.1 \\
\hline 11 & 2 & 46.7 & 15.2 & 2 & $1,386.8$ & 205.9 & 3 & $3,713.6$ & 622.8 \\
\hline 12 & 2 & 63.7 & 11 & 3 & $1,622.9$ & 204.5 & 3 & $4,339.6$ & 612.7 \\
\hline 13 & 2 & 50.3 & 13 & 3 & $1,584.7$ & 205.1 & 3 & $4,078.3$ & 614.6 \\
\hline 14 & 2 & 49.2 & 13 & 3 & 1,396 & 206.8 & 3 & $4,296.1$ & 613.9 \\
\hline 15 & 3 & 59.2 & 12.7 & 3 & $1,362.2$ & 207.2 & 3 & $4,032.6$ & 617.5 \\
\hline 16 & 3 & 66.5 & 12.2 & 3 & $1,312.5$ & 216.3 & 3 & $4,065.8$ & 615.1 \\
\hline 17 & 3 & 60.4 & 12.3 & 3 & $1,515.1$ & 206.1 & 3 & $3,632.8$ & 672.2 \\
\hline 18 & 3 & 55.3 & 13.1 & 3 & $1,424.9$ & 206.3 & 3 & $4,041.2$ & 616.8 \\
\hline 19 & 3 & 56.8 & 12.9 & 3 & $1,455.1$ & 206.2 & & & \\
\hline 20 & 3 & 48.9 & 15.2 & 3 & $1,334.8$ & 210.3 & & & \\
\hline 21 & 3 & 51.2 & 14.3 & & & 205.2 & & & \\
\hline
\end{tabular}




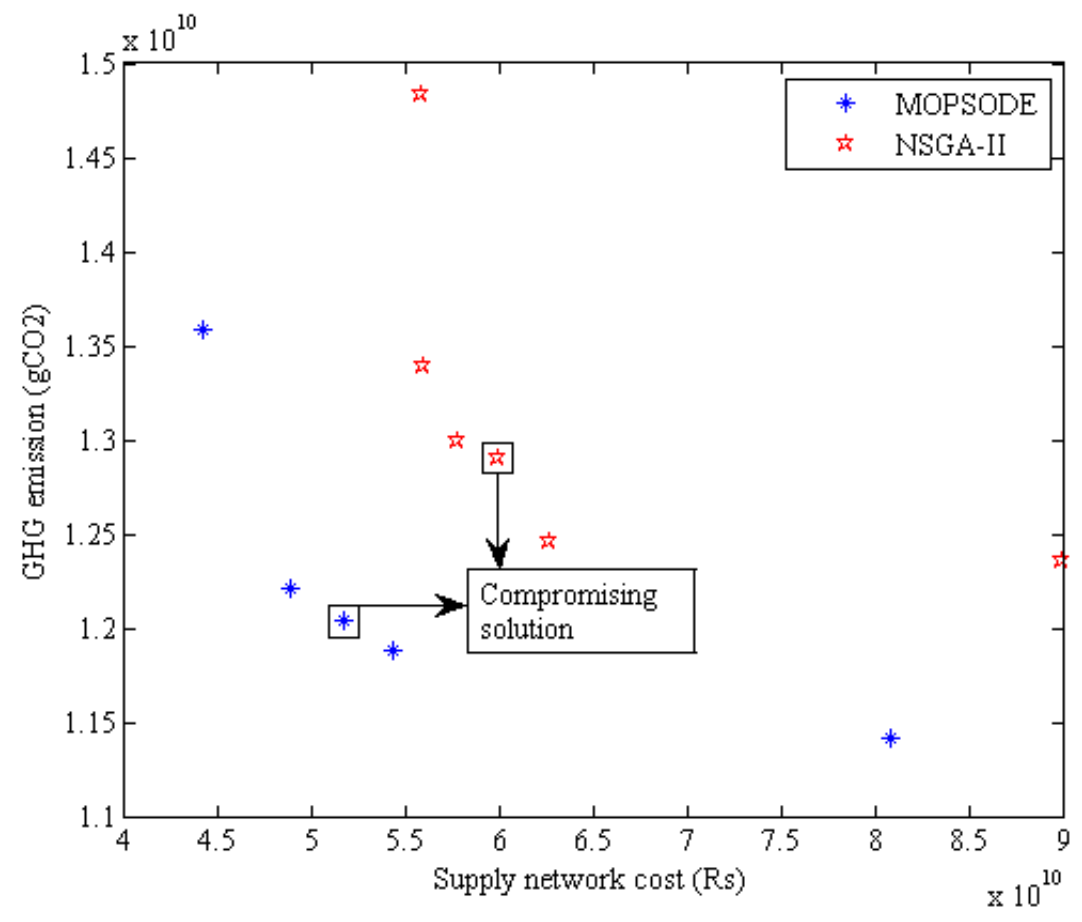

Fig. 6 (a). Pareto dominance of MOPSODE over NSGA-II for small data set (5-3-3-5-2)

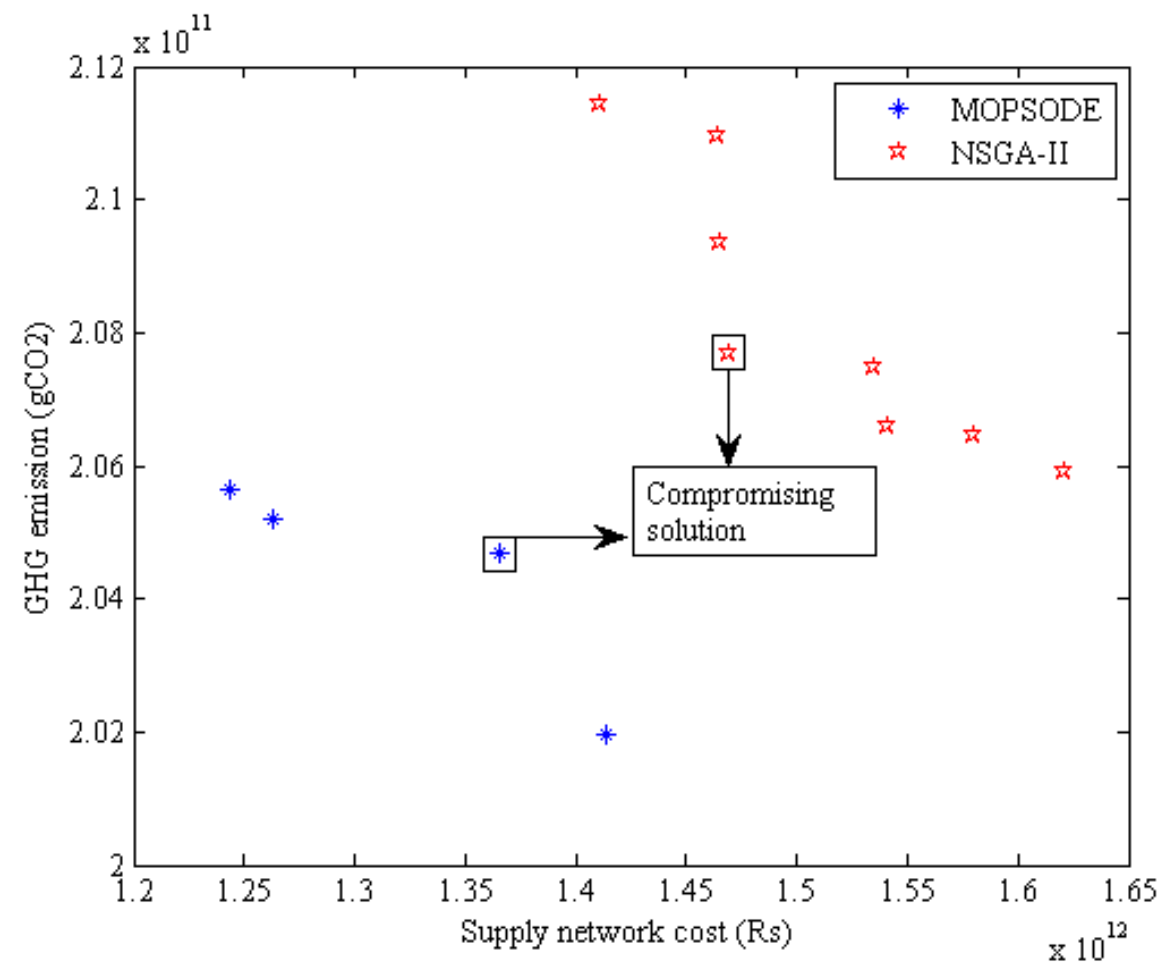

Fig. 6 (b). Pareto dominance of MOPSODE over NSGA-II for medium data set (6-3-5-9-3) 


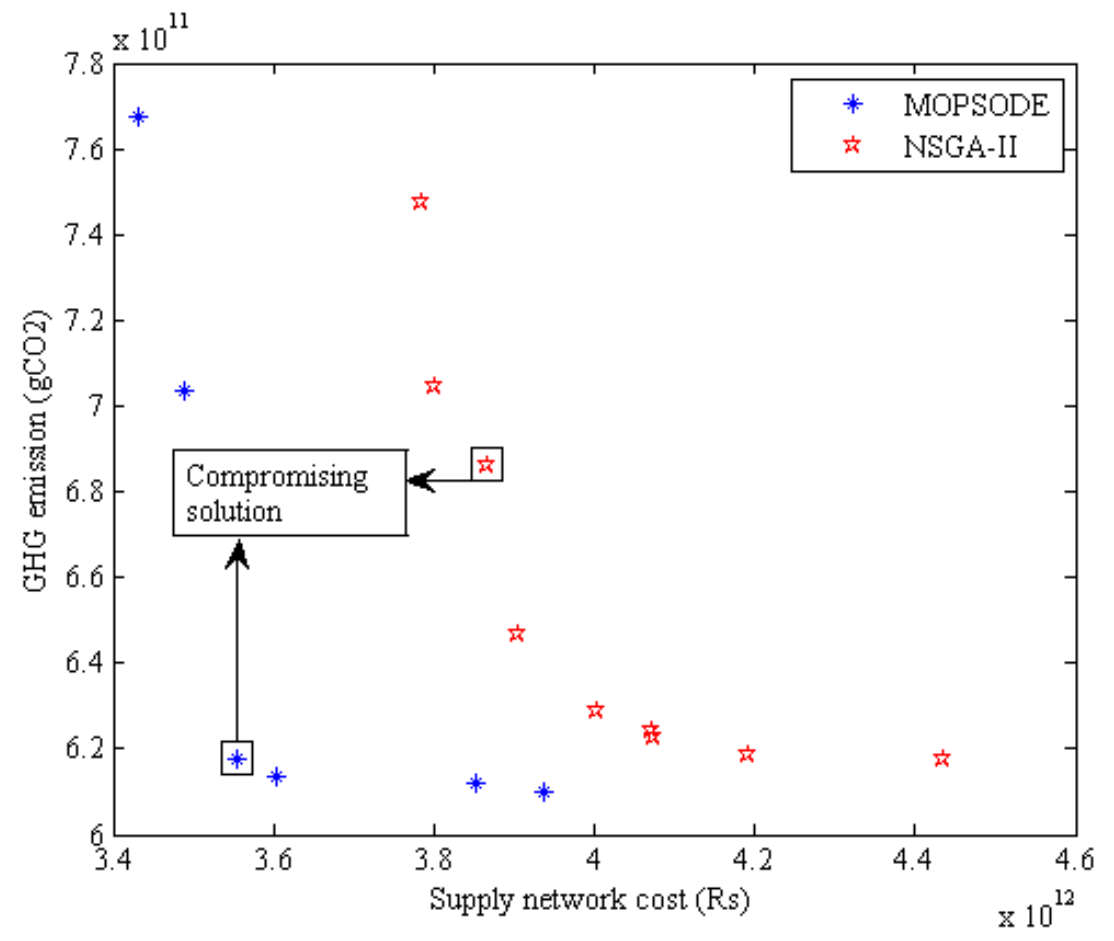

Fig. 6 (c). Pareto dominance of MOPSODE over NSGA-II for large data set (10-4-4-10-3)

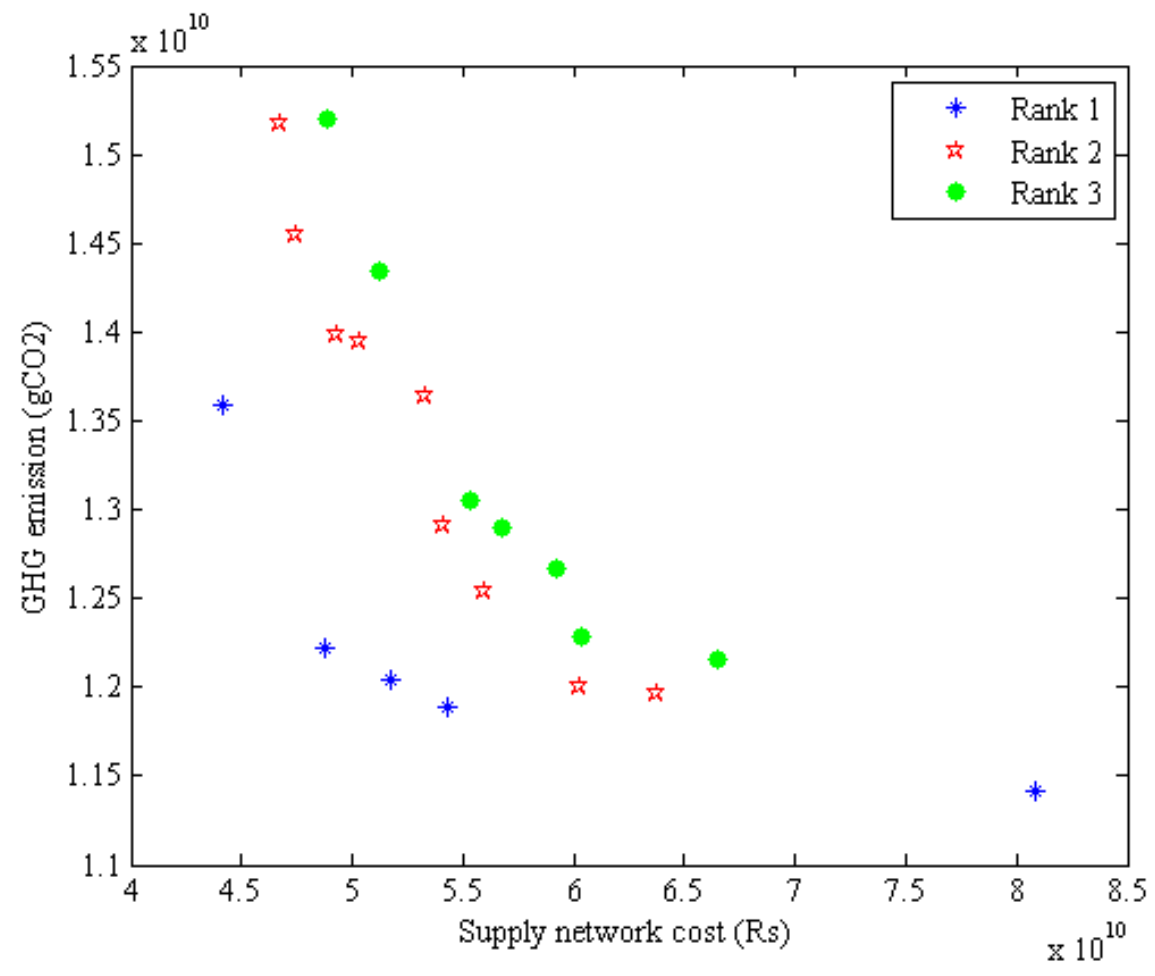

Fig. 7 (a). Pareto optimal fronts obtained using MOPSODE for small data set (5-3-3-5-2) 


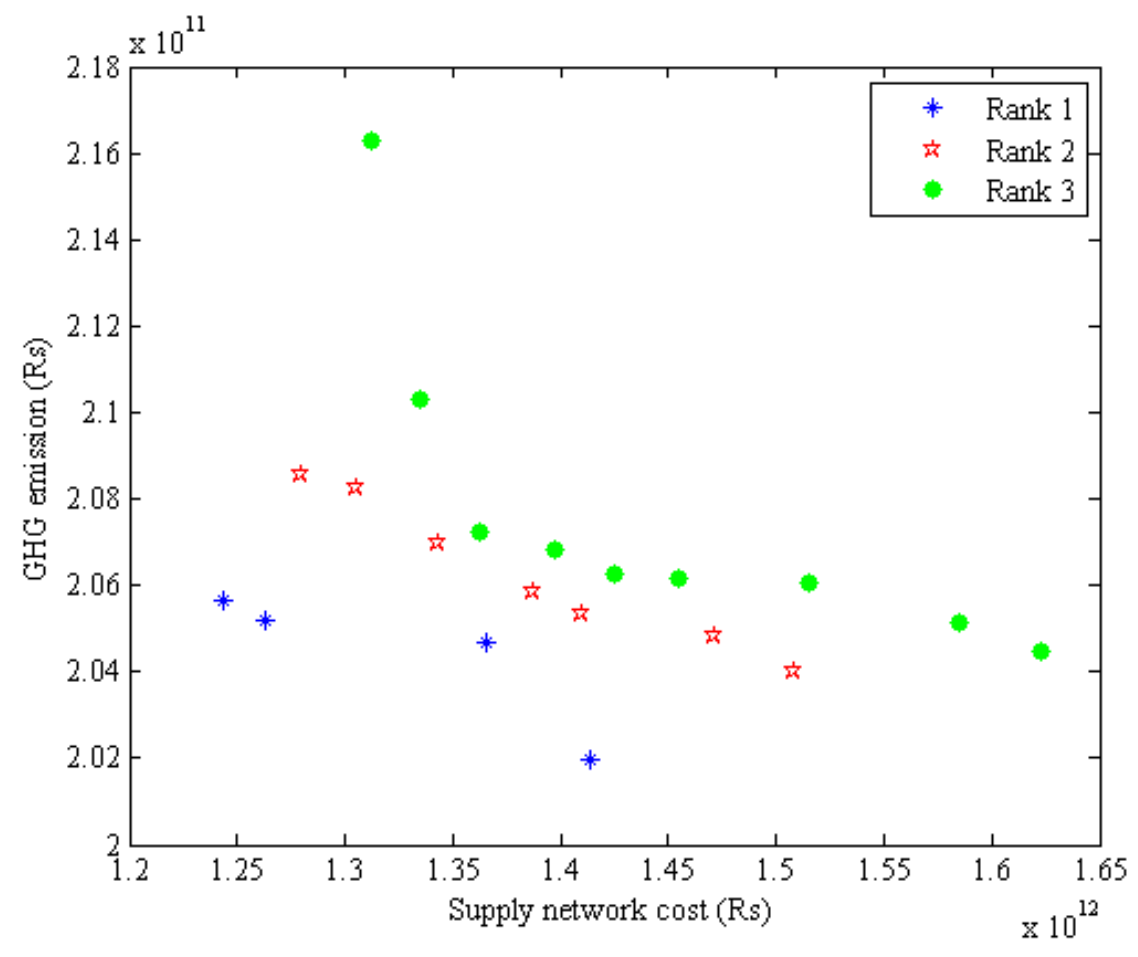

Fig. 7 (b). Pareto optimal fronts obtained using MOPSODE for medium data set (6-3-5-9-3)

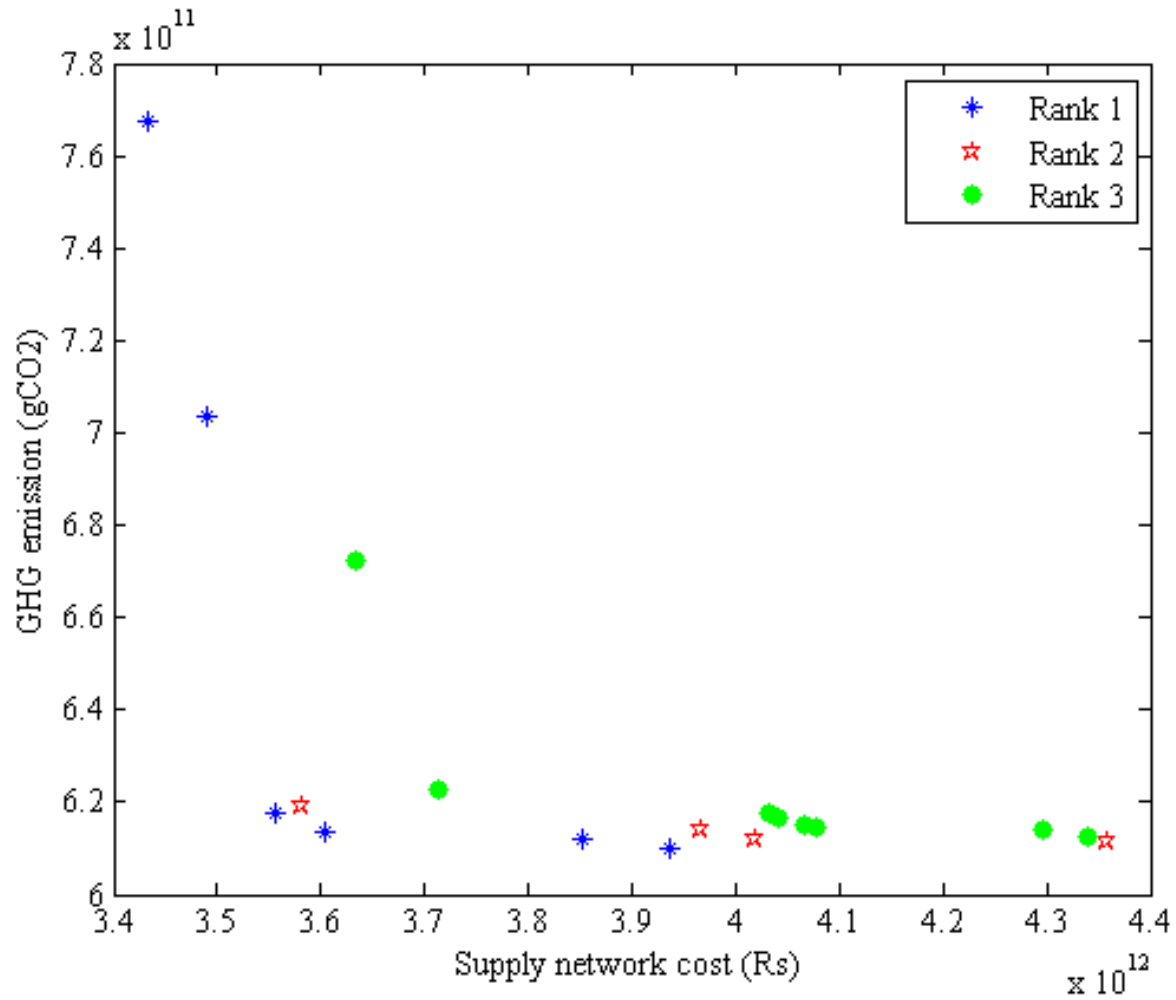

Fig. 7 (c). Pareto optimal fronts obtained using MOPSODE for large data set (10-4-4-10-3) 
The summary of decision variables for the given instances of the problem are presented in Tables H.1-H.5. Table H.1 summarizes the region-wise food grain shipment quantities, route selection and transportation mode selection between the origin and destination states for all the problem sets. The small data set consists of 3 regions and in the origin as well as the destination state (refer Appendix E). For small dataset, it is observed that selected quantity of shipments that minimize total supply network cost and GHG missions are majorly moved from origin regions 1 and 2 to all regions in the destination state as compared to the shipments from the third origin region. It is important to note that when there is higher quantity of transport involved, rail dominates the mode of transport indicated by last two columns of Table H.1. On the contrary, road mode of transport is majorly selected for smaller shipment sizes. Table H.2 presents the frequency and type of rail/road transport selected for realizing the defined shipments with in the origin state. The number of intermodal hubs $(k)$ in the origin state for small and medium problems is 3 and for the large size is 4 (refer Appendix E). A value of zero in columns (4-11) of Table H.2 indicates that there is no route that corresponds to rail or road transport, whereas an integer value greater than zero represents the number of routes by rail or road, directed towards origin intermodal hubs from each origin region. The last four columns represent the total number of trucks or rakes used for transportation in this segment. It is observed that in some cases, there is no rail or road selected for transport because of no movement of food grains within state from the corresponding region to the origin intermodal hub. A similar interpretation could be made for the transportation phenomenon in the destination state from Tables H.3 and H.4. Finally, Table H.5 provides description of rake allocation summary for the transport segment between origin and destination intermodal hubs along with the destination hubs allocated for all datasets. Further to this, it is observed that all the origin intermodal potential hubs are selected to be opened whereas the 
potential destination hubs, remained close in two instances for small dataset, three instances for medium and large datasets.

The results obtained by MOPSODE converge faster when compared to NSGA-II because of the faster convergence speed of embedded particle swarm optimization. In agreement with this, the velocity of the particle was found to be proportional to the size of problem indicating good performance (Domínguez et al. 2011) by MOPSODE. The convergence metric called generational distance (GD) employed by Su and Chi (2017) was adopted to evaluate the performance of the proposed metaheuristic. Generational distance measures the distance of the current pareto front obtained with respect to a given near optimal pareto front. It was observed that over a span of 20 trial runs, the GD was found to vary with less than $1 \%$ deviation for all the problem sets which confirms the near optimality of the resulting pareto fronts. The graphical illustration of the near optimal solution obtained for small data set is shown in Fig. G.1.

\subsection{Impact of variation in hub location level}

As presented earlier in section 4, the current formulation incorporates the possibility of restricting the number of hubs located in a given instance of the problem. Haphazard selection of hub location level may prove to be beneficial in some cases, but detrimental in majority of the cases to broader objectives of the problem. Therefore, it is important to maintain an adequate level of hub location to ensure optimal flow of food grains. In this paper, the impact of closing the intermodal hubs on supply network cost and GHG emission is captured by hub closure impact index, I evaluated as, $I=1-\left(C_{0} / C\right)$, where $C$ is the average non-dominated total cost for a given configuration of hub location. $C_{0}$ is the average non-dominated total cost for the case with zero number of hub closures. The effect of level variations in destination intermodal hubs located while keeping the origin hub location level fixed on supply network cost and GHG emission is investigated through set of 
experiments shown in Table 5. Fig. 8 shows the rank 1 pareto fronts obtained for different types of experiments conducted. The results shown in Table 5 and Fig. 8 correspond to medium size data set from where it can be verified that the value of $I$ increases as the hub location level decreases and is found to be the highest for experiment 4 ( 3 destination hubs closed) indicating the increasing level of impact on the overall cost with corresponding increase in the level of hub closures, following which, $I$ is found to deteriorate for experiment 5 (4 destination hubs closed). This reduction in the value of $I$ represents the sudden drop in the level of transport due to lower levels of food grain demand resulting from demand loss associated with closed hubs. On this basis, it is important that the decision maker choses a suitable level of hub location while adjusting the tradeoff between demands lost due to hubs closed with the overall cost.

Table 5 Impact of hub closure on cost and emission

\begin{tabular}{|c|c|c|c|c|c|}
\hline & \multicolumn{5}{|c|}{ Experiment } \\
\hline & 1 & 2 & 3 & 4 & 5 \\
\hline $\begin{array}{l}\text { Origin hub location } \\
\text { level }\left(b_{o}\right)\end{array}$ & 3 & 3 & 3 & 3 & 3 \\
\hline $\begin{array}{l}\text { Destination hub } \\
\text { location level }\left(b_{d}\right)\end{array}$ & 5 & 4 & 3 & 2 & 1 \\
\hline $\begin{array}{l}\text { No. of destination hubs } \\
\text { closed }\end{array}$ & 0 & 1 & 2 & 3 & 4 \\
\hline $\begin{array}{l}\text { Supply network cost } \\
\text { (bn Rs) }\end{array}$ & 294.9 & $1,365.6$ & $2,233.5$ & $2,805.6$ & $2,314.3$ \\
\hline $\begin{array}{l}\text { GHG emission } \\
\left(\mathrm{kt} \mathrm{CO}_{2}\right)\end{array}$ & 191.3 & 204.7 & 213.8 & 388.9 & 203.3 \\
\hline $\begin{array}{l}\text { Average non- } \\
\text { dominated total cost } \\
\text { (bn Rs) }\end{array}$ & 321.8 & $1,319.5$ & $2,295.6$ & $3,213.2$ & 2,380 \\
\hline $\begin{array}{l}\text { Hub closure impact } \\
\text { index }\end{array}$ & 0 & 0.76 & 0.86 & 0.9 & 0.87 \\
\hline
\end{tabular}




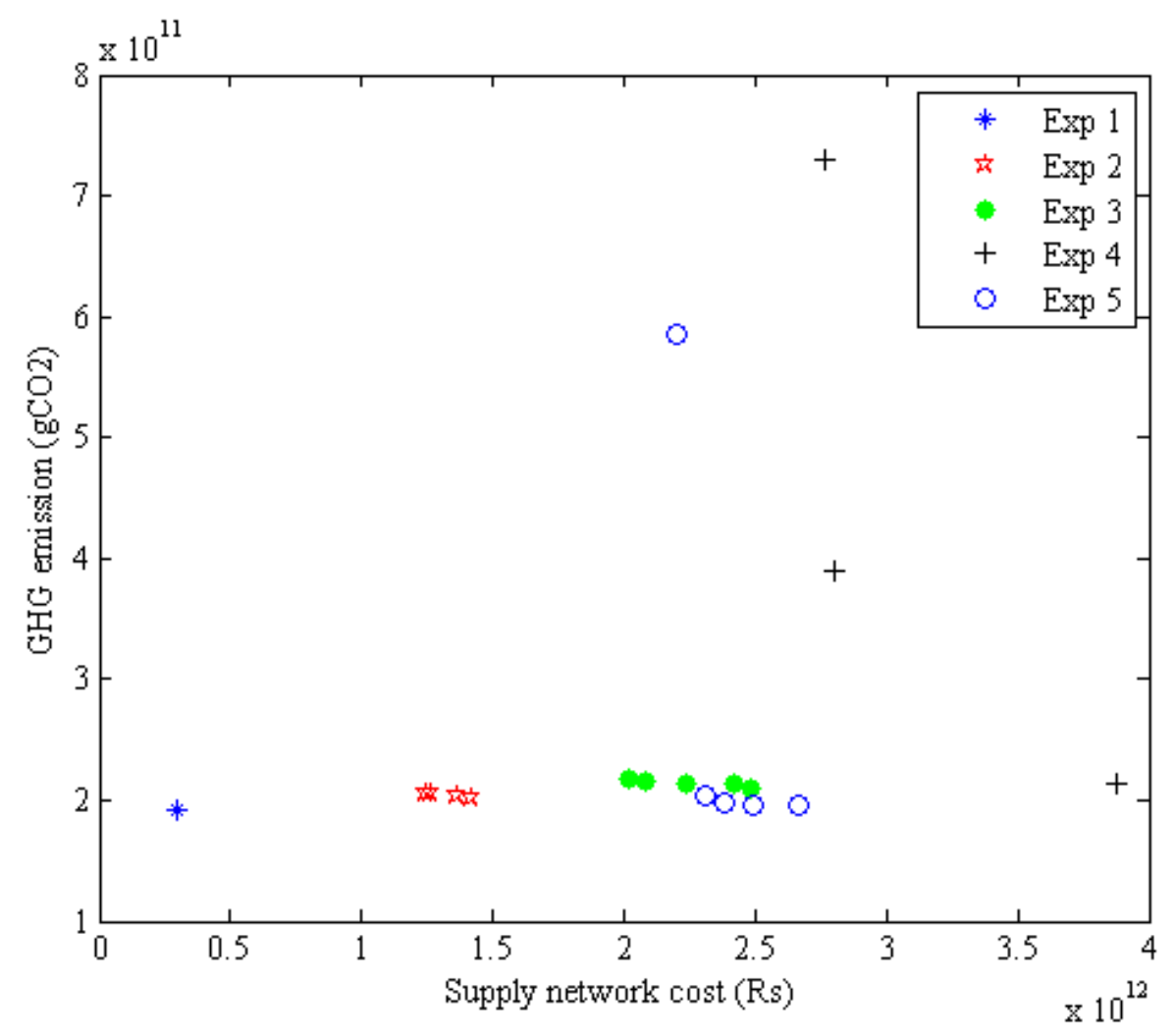

Fig. 8. Pareto fronts generated while investigating the impact of varying hub location level

\subsection{Impact of variation in food grain wastage threshold}

This paper attempts to restrict the overall food grain wastages by setting a threshold on intermodal facility level wastages while realizing the total shipment quantity from origin to destination warehouses. Five experiments with distinct food grain wastage threshold levels described as earlier in section 6, while keeping remaining parameters fixed are conducted on medium size data set to explore the effect of their changing levels on the supply network cost and GHG emission. The first experiment corresponds to the highest wastage threshold setting and the subsequent experiments are conducted for decreasing level of wastage threshold settings as shown in Table F.3. Table 6 records the maximum and minimum supply network cost and GHG emission observed for experiments conducted on medium size dataset with the aforementioned settings. Observations reveal that reducing the wastage threshold up to $50 \%$ causes reduction in supply network cost at 
the cost of increasing levels of GHG emission. Further increasing the wastage threshold was found to cause damage to both supply network cost and GHG emission levels. Enforcing further restriction on the wastage cap generated only infeasible solutions. Fig. 8 captures rank 1 pareto optimal fronts for the each of the five experiments.

Table 6 Effect of food grain wastage threshold on cost and emission

\begin{tabular}{llllll}
\hline Experiment & $\begin{array}{l}\text { Food grain } \\
\text { wastage } \\
\text { threshold level }\end{array}$ & $\begin{array}{l}\text { Minimum } \\
\text { supply network } \\
\text { cost (bn Rs) }\end{array}$ & $\begin{array}{l}\text { Maximum } \\
\text { supply network } \\
\text { cost (bn Rs) }\end{array}$ & $\begin{array}{l}\text { Minimum GHG } \\
\text { emission } \\
(\mathrm{kt} \mathrm{CO})\end{array}$ & $\begin{array}{l}\text { Maximum } \\
\mathrm{GHG} \mathrm{emission})\end{array}$ \\
\hline 1 & 1 & $1,243.3$ & 1,413 & 201 & 205.7 \\
2 & 2 & $1,110.9$ & $1,370.2$ & 203.8 & 205.7 \\
3 & 3 & 772 & $1,102.5$ & 200.8 & 262.6 \\
4 & 4 & $1,204.9$ & $1,554.5$ & 204.8 & 210.4 \\
5 & 5 & $1,354.4$ & $1,645.4$ & 206 & 218.8 \\
\hline
\end{tabular}

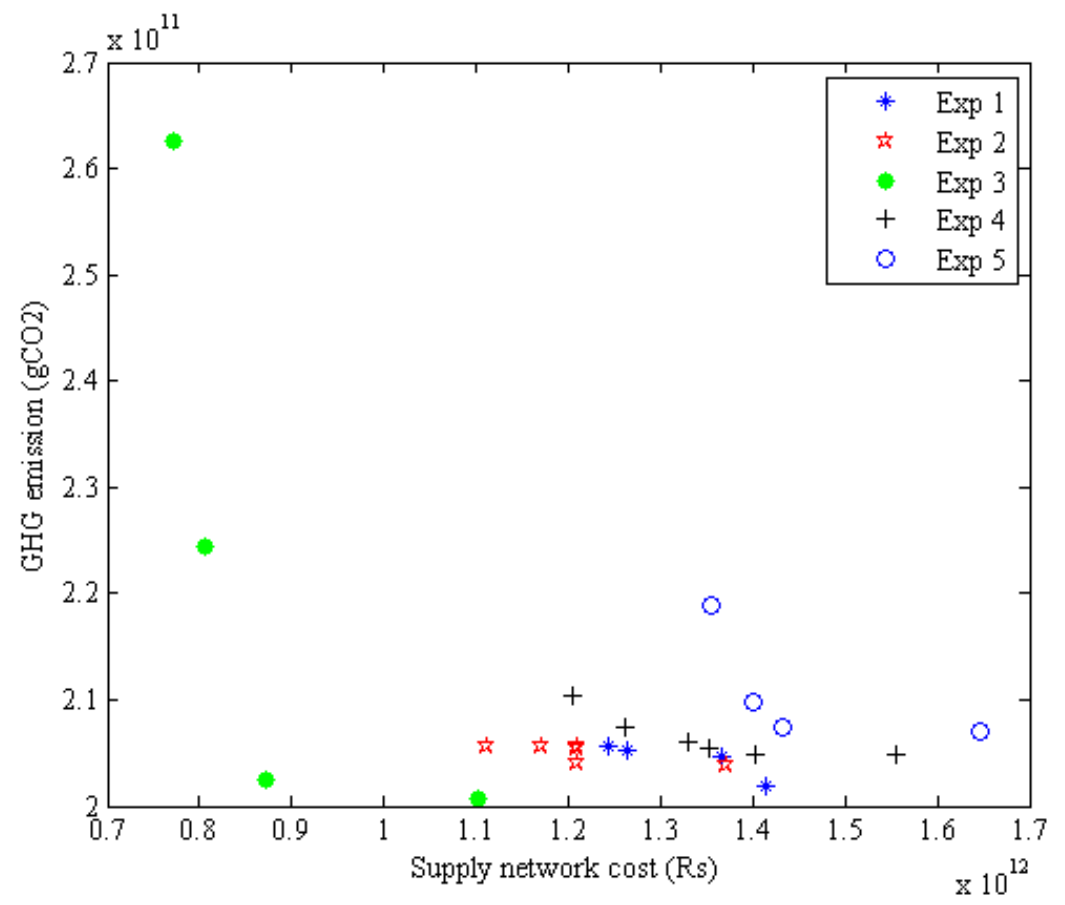

Fig. 8. Pareto fronts generated while investigating the impact of varying food grain wastage threshold 


\subsection{Impact of variation in intermodal hub capacity}

The level of intermodal hub capacities regulate the flow of food grains routed through the intermodal hubs. High capacity levels ensure routing of shipments through low cost hubs. However, in practical scenarios, some portion of the capacity often remains unutilized due to multiple reasons. In such cases, it becomes necessary to investigate the effect of different intermodal hub capacity levels in the presence of wastages on the supply network cost and GHG emission to recognize alternative solutions with subtle variation in cost and emission. To this end, four experiments with distinct hub capacity levels (Table F.3) are conducted while keeping all other parameters as fixed. Results indicate that for the given instance (medium size data set)

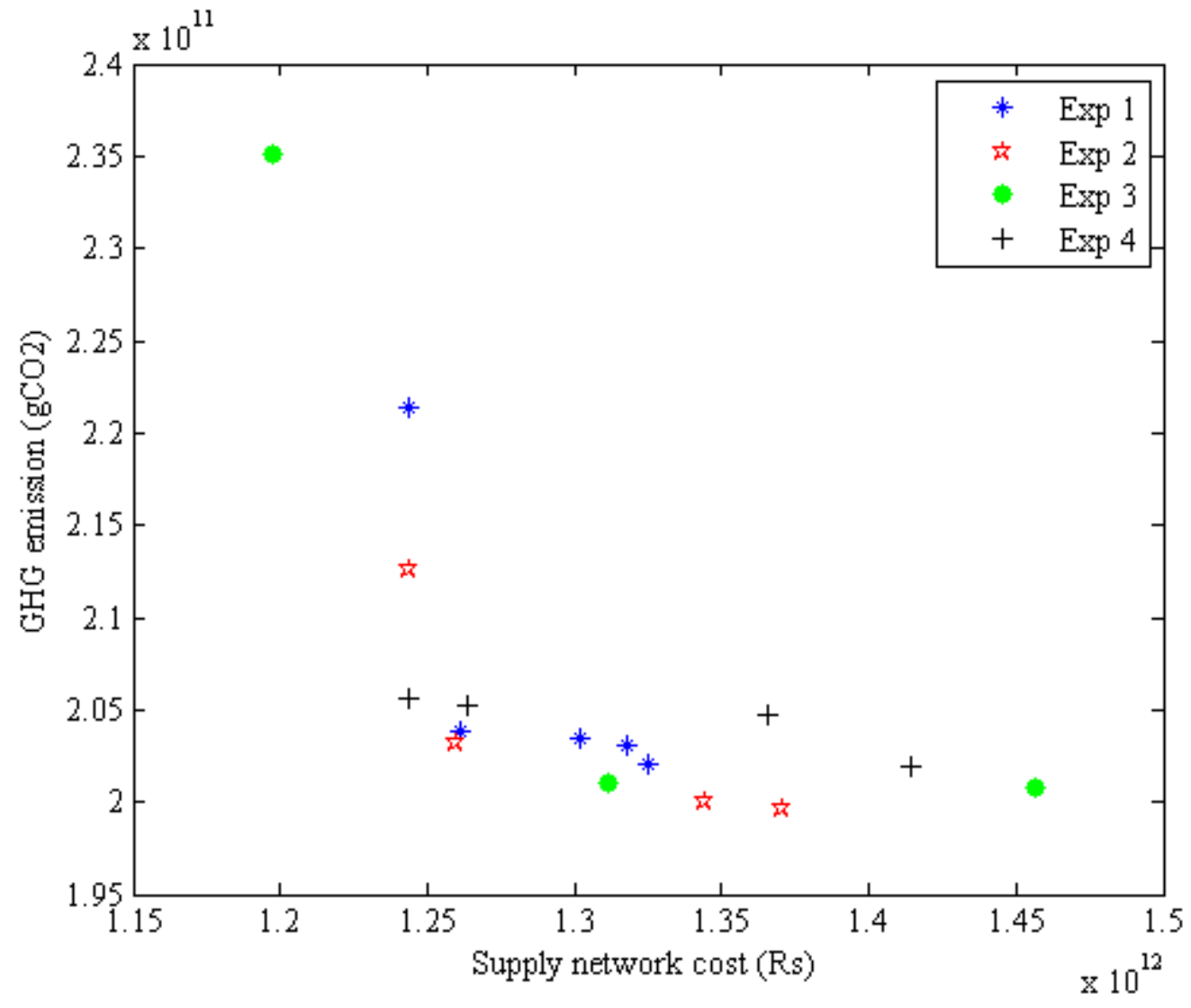

Fig. 9. Pareto fronts generated while investigating the effect of varying intermodal hub capacity 
increasing the origin and destination hub capacities (experiments 2 and 3) individually was found to give a better spread of solution trade-offs (Table 7) but with lesser number of good compromise solutions (Fig. 9), while, maintaining the origin and destination hubs at higher capacity levels simultaneously (experiment 4) ensured reduced supply network costs at lower levels of GHG emission. Table 7 lists the pareto front boundaries in terms of maximum and minimum level realizations of both objectives for this study. Fig. 9 displays the optimal pareto fronts obtained for experiments 1-4 pertaining to this study.

Table 7 Effect of intermodal hub capacity on cost and emission

\begin{tabular}{llllll}
\hline $\begin{array}{l}\text { Experi- } \\
\text { ment }\end{array}$ & $\begin{array}{l}\text { Intermodal } \\
\text { hub capacity } \\
\text { level }\end{array}$ & $\begin{array}{l}\text { Minimum supply } \\
\text { network } \\
\text { cost (bn Rs) }\end{array}$ & $\begin{array}{l}\text { Maximum } \\
\text { supply network } \\
\text { cost (bn Rs) }\end{array}$ & $\begin{array}{l}\text { Minimum GHG } \\
\text { emission } \\
\left(\mathrm{kt} \mathrm{CO}_{2}\right)\end{array}$ & $\begin{array}{l}\text { Maximum GHG } \\
\text { emission } \\
\left(\mathrm{kt} \mathrm{CO}_{2}\right)\end{array}$ \\
\hline 1 & 1 & $1,244.1$ & 1,325 & 202.1 & 221.4 \\
2 & 2 & $1,244.1$ & $1,370.7$ & 199.7 & 212.6 \\
3 & 3 & $1,197.1$ & $1,456.5$ & 200.8 & 235.1 \\
4 & 4 & $1,243.3$ & 1,413 & 201 & 205.7 \\
\hline
\end{tabular}

Table 8 Decrease in GHG emission versus increase in supply network cost for all problem sets

\begin{tabular}{lllll}
\hline $\begin{array}{l}\text { Problem } \\
\text { set }\end{array}$ & $\begin{array}{l}\text { Supply network } \\
\text { cost (bn Rs) }\end{array}$ & $\begin{array}{l}\text { GHG emissions } \\
\left(\mathrm{kt} \mathrm{CO}_{2}\right)\end{array}$ & $\begin{array}{l}\text { Percentage } \\
\text { decrease in GHG } \\
\text { emissions }\end{array}$ & $\begin{array}{l}\text { Percentage increase in } \\
\text { supply network cost }\end{array}$ \\
\hline Small & 44.1 & 13.6 & - & - \\
& 48.8 & 12.2 & 10.090 & 10.622 \\
& 51.7 & 12 & 1.387 & 5.933 \\
& 54.3 & 11.9 & 1.326 & 4.979 \\
& 80.8 & 11.4 & 3.979 & 48.916 \\
\hline Medium & $1,243.3$ & 205.7 & - & - \\
& $1,263.4$ & 205.2 & 0.231 & 1.609 \\
& $1,365.6$ & 204.7 & 0.245 & 8.090 \\
& 1,413 & 201 & 1.323 & 3.546 \\
\hline Large & 1,413 & 592.5 & - & - \\
& $1,422.7$ & 592.2 & 0.038 & 0.685 \\
& $1,436.8$ & 592.1 & 0.056 & 1.686 \\
\hline
\end{tabular}




\section{Conclusion}

This paper provides an effective decision support tool for solving environmentally focused real time food grain transportation problem considering wastages at the facility level with minimization of total supply network cost and GHG emission as a bi-objective formulation. The unique contributions of this work are three fold. Firstly, a mixed integer environmentally conscious, wastage aware and economically convenient non-linear optimization model is formulated with simultaneous consideration towards economic and environmental objectives in the food grain transportation domain. In this regard, the idea of reducing food grain wastages is mathematically captured at intermodal facility level while restricting the total food grain wastage within a specified threshold. The model adequately captures real time transportation constraints with respect to vehicle capacities, intermodal hub capacity restrictions, multi-modal considerations and available inventory in addition to food grain wastages. Secondly, a modified version of multi-objective particle swarm optimization with differential evolution algorithm with a new penalty allocation scheme is proposed to tackle the computationally complex constrained optimization problem. Thirdly, a hub closure impact index is proposed to quantify the impact of hub closures on economic and environmental objectives. The proposed model is tested on three differently sized problem instances inspired from food grain industry in Indian context. Benchmarking results validate the superiority of MOPSODE over NSGA-II for small, medium and large instances of the problem with subtle variations in computational time. Subsequently, the importance of identifying compromising solution is illustrated by examining the trade-offs through pareto analysis in relevance to the considered instances. Specific insights are developed through sensitivity analysis to explore the effect of changing levels of hub location level, food grain wastage threshold and intermodal hub capacities on the supply network cost and GHG emission. Observations reveal that 
significant amount of wastage reduction is achieved at an expense of minimal increase in the total supply network cost much to the interest of decision makes and stakeholders.

The managerial implications with respect to the cost-emission tradeoffs observed for various configurations of the current problem are derived as follows. First, for the small and medium size problem (Fig. 7 (a) and Fig. 7 (c)), the rate of reduction in total GHG emission with respect to increase in the total supply network cost is found to increase initially and later decrease drastically. Whereas, for the medium case (Fig. 7 (b)), the rate of reduction is found to decrease initially and later increase. Table 8 summarizes the percentage decrease in GHG emissions achieved against the corresponding percentage increase in total supply network costs. Second, the insights gained by capturing the impact of variation in hub location level on the supply network cost and GHG emission are of crucial value to managers for deciding the adequate level of hub location. It is recommended that hub location level must be kept lower than the breakeven point that defines the tradeoff between demands lost due to hubs closed and the overall cost. Further, this model helps the stakeholders in staying well informed with respect to the achievable levels of wastage thresholds while ensuring the check on cost and emission. The paper provides numerical evidence to showcase pros and cons of augmenting the intermodal hub capacities. The aforementioned trade-offs are instrumental for stakeholders and decision makers while making an intuitive choice of sustainable strategy for food grain shipments considering wastages.

The current work holds immense potential to be extended towards strengthening the triple bottom line approach by considering social objectives in terms of customer and employee satisfaction. Further, exploring the effect of quality degradation especially with respect to wheat and other perishable food grain products remains a necessary and important open research question. The current formulation can be further enriched by incorporating deterministic and probabilistic 
disruptions while being addressed by enhanced decomposition and relaxation schemes embedded with evolutionary optimization.

\section{Acknowledgement}

This research is conducted in the context of project titled, 'Transportation management to implement NFSA (TMI)' sponsored by Ministry of Human Resource and Development (MHRD), Department of Higher Education, Government of India [sanction letter No. F. NO. 4-25/2013-TSI]. The authors are sincerely thankful to the project coordinator, Prof. M. K. Tiwari, (Department of Industrial \& Systems Engineering, Indian Institute of Kharagpur, West Bengal, India) for providing his timely support and valuable insights througout the course of this research. We express special gratitude to FCI authorites to faciliate the conduct of field study which has significantly contributed to the understanding of Indian food grain transportation operations.

\section{Appendix A. Symbols and Notations}

Indices

\begin{tabular}{ll}
$o$ & Origin state \\
$d$ & Destination state \\
$i$ & Origin FCI warehouse \\
$j$ & Destination FCI warehouse \\
$p$ & Origin region \\
$q$ & Destination region \\
$k$ & Origin hub \\
$m$ & Destination hub \\
$\phi$ & Road \\
$\psi$ & Rail/Rake \\
$t$ & Time period \\
Sets & \\
\hline
\end{tabular}


$R_{o} \quad$ Set of regions in origin state $o$

$R_{d} \quad$ Set of regions in destination state $d$

$W_{p} \quad$ Set of FCI warehouses in origin region $p$

$W_{q} \quad$ Set of FCI warehouses in destination region $q$

$H_{o} \quad$ Set of potential hub locations in origin state $o, H_{o} \subset \bigcup_{p=1}^{\left|R_{o}\right|} W_{p}$

$H_{d} \quad$ Set of potential hub locations in destination state $d, H_{d} \subset \bigcup_{q=1}^{\left|R_{d}\right|} W_{q}$

$T \quad$ Set of time periods

Parameters

$C_{i p k}^{o \phi} \quad$ Unit cost of transportation by road from origin warehouse $i$ of region $p$ to origin hub $k$ where, $i \in W_{p}, p \in R_{o}, k \in H_{o}$

$C_{i p k}^{o \psi} \quad$ Unit cost of transportation by rail from origin warehouse $i$ of region $p$ to origin hub $k$, where, $i \in W_{p}, p \in R_{o}, k \in H_{o}$

$C_{k m}^{o d} \quad$ Unit cost of transportation by rail from origin hub $k$ to destination hub $m$, where, $k \in H_{o}, m \in H_{d}$

$C_{m j q}^{d \phi} \quad$ Unit cost of transportation by road from destination hub $m$ to warehouse $j$ of region $q$, where, $m \in H_{d}, j \in W_{q}, q \in R_{d}$

$C_{m j q}^{d \psi} \quad$ Unit cost of transportation by rail from destination hub $m$ to warehouse $j$ of region $q$, where, $m \in H_{d}, j \in W_{q}, q \in R_{d}$

$C_{i p k m t}^{j q} \quad$ Unit cost of total transport from warehouse $i$ of region $p$ to warehouse $j$ of region $q$ through origin hub $k$ and destination hub $m$ in time period $t$, where, $i \in W_{p}, p \in R_{o}$, $j \in W_{q}, q \in R_{d}, k \in H_{o}, m \in H_{d}, t \in T$

$A_{k t}^{o} \quad$ Cost of locating warehouse facility at origin hub $k$ in time period $t$, where, $k \in H_{o}$, $t \in T$

$A_{m t}^{d} \quad$ Cost of locating warehouse facility at origin hub $m$ in time period $t$, where, $m \in H_{d}$, $t \in T$ 
$C_{h} \quad$ Unit cost of handling food grains at intermodal hub

$C_{p} \quad$ Unit penalty cost for violating service time restrictions

$a_{i p k}^{o \phi} \quad$ Road distance from warehouse $i$ of region $p$ to origin hub $k$, where $i \in W_{p}, p \in R_{o}$, $k \in H_{o}$

$a_{i p k}^{o \psi} \quad$ Rail distance from warehouse $i$ of region $p$ to origin hub $k$, where $i \in W_{p}, p \in R_{o}$, $k \in H_{o}$

$a_{k m}^{o d} \quad$ Rail distance from origin hub $k$ to destination hub $m$, where $k \in H_{o}, m \in H_{d}$

$a_{m j q}^{d \phi} \quad$ Road distance from destination hub $m$ to warehouse $j$ of region $q$, where $m \in H_{d}$, $j \in W_{q}, q \in R_{d}$

$a_{m j q}^{d \psi} \quad$ Rail distance from destination hub $m$ to warehouse $j$ of region $q$, where $m \in H_{d}$, $j \in W_{q}, q \in R_{d}$

$\tau_{j q t} \quad$ Service time upper limit for the food grain demand at warehouse $j$ of region $q$, where $j \in W_{q}, q \in R_{d}$

$\alpha \quad$ Transportation cost consolidation factor

$\beta \quad$ Travel time consolidation factor

$D_{q t} \quad$ Demand for food grains at region $q$ in time period $t$, where, $q \in R_{d}, t \in T$

$I_{i p 0}$ Initial food grain inventory (inventory at the end of $t=0$ ) at warehouse $i$ of region $p$, where, $i \in W_{p}, p \in R_{o}$

$P_{i p t} \quad$ Food grain procurement at warehouse $i$ of region $p$ realized in time period $t$, where, $i \in W_{p}, p \in R_{o}, t \in T$

$b_{o} \quad$ Number of origin hubs allowed to open in any time period.

$b_{d} \quad$ Number of destination hubs allowed to open in any time period.

$U_{k} \quad$ Capacity of origin hub $k$, where, $k \in H_{o}$

$U_{m} \quad$ Capacity of destination hub $m$, where, $m \in H_{d}$

$\mu_{o k} \quad$ Fraction of food grain quantity lost at origin hub $k$, where, $k \in H_{o}$ 
$\mu_{d m} \quad$ Fraction of food grain quantity lost at destination hub $m$, where, $m \in H_{d}$

$C_{\phi} \quad$ Unit truck load capacity

$C_{\psi} \quad$ Unit train load capacity

$\rho_{i p t}^{o \phi} \quad$ Number of trucks available for loading in origin warehouse $i$ of region $p$ in time period $t$, where, $i \in W_{p}, p \in R_{o}, t \in T$

$\rho_{i p t}^{o w} \quad$ Number of rakes available for loading in origin warehouse $i$ of region $p$ in time period $t$, where, $i \in W_{p}, p \in R_{o}, t \in T$

$\sigma_{k t} \quad$ Number of rakes available in origin hub $k$ in time period $t$, where, $k \in H_{o}, t \in T$

$\rho_{m t}^{d \phi} \quad$ Number of trucks available for loading in destination hub $m$ in time period $t$, where, $m \in H_{d}, t \in T$

$\rho_{m t}^{d \psi} \quad$ Number of rakes available for loading in destination hub $m$ in time period $t$, where, $m \in H_{d}, t \in T$

$\varepsilon_{i p} \quad=1$, if warehouse $i$ of region $p$ is a potential hub, where $i \in W_{p}, p \in R_{o}, 0$ otherwise

$M \quad$ Large number

Decision variables

$x_{i p k m t}^{j q} \quad$ Quantity of food grains to be dispatched from origin warehouse $i$ of region $p$ towards destination warehouse $j$ of region $q$ through origin hub $k$ and destination hub $m$ in time period $t$, where, $i \in W_{p}, p \in R_{o}, j \in W_{q}, q \in R_{d}, k \in H_{o}, m \in H_{d}, t \in T$

$I_{i p t} \quad$ Food grain inventory available at warehouse $i$ of region $p$ at the end of time period $t$, where, $i \in W_{p}, p \in R_{o}, t \in T$

$l_{i p k t} \quad$ Number of trucks or rakes used to transport food grains from warehouse $i$ of region $p$ to hub $k$ in time period $t$, where, $i \in W_{p}, p \in R_{o}, k \in H_{o}, t \in T$

$n_{k m t} \quad$ Number of rakes used to transport food grains from origin hub $k$ to destination hub $m$ in time period $t$, where, $k \in H_{o}, m \in H_{d}, t \in T$ 
$h_{\text {mjqt }} \quad$ Number of trucks or rakes used to transport food grains from destination hub $m$ to destination warehouse $j$ of region $q$ in time period $t$, where, $j \in W_{q}, q \in R_{d}, m \in H_{d}$, $t \in T, 0$ otherwise

$y_{i p k m t}^{j q}=1$, if there is positive quantity of flow from origin warehouse $i$ of region $p$ to destination warehouse $j$ of region $q$ through origin hub $k$ and destination hub $m$ in time period $t$, where, $i \in W_{p}, p \in R_{o}, j \in W_{q}, q \in R_{d}, k \in H_{o}, m \in H_{d}, t \in T, 0$ otherwise

$\delta_{i p k t}^{o \phi} \quad=1$, if road is selected as mode of transport for shipping the quantity, $x_{i p k m t}^{j q}$ from origin warehouse $i$ of region $p$ to origin hub $k$ in time period $t$, where, $i \in W_{p}, p \in R_{o}$, $k \in H_{o}, t \in T, 0$ otherwise

$\pi_{i p t}^{o \phi} \quad=1$, if road is selected as mode of transport for shipments starting from origin warehouse $i$ of region $p$ in time period $t$, where, $i \in W_{p}, p \in R_{o}, t \in T, 0$ otherwise

$\delta_{i p k t}^{o \psi} \quad=1$, if rail is selected as mode of transport for shipping the quantity, $x_{i p k m t}^{j q}$ from origin warehouse $i$ of region $p$ to origin hub $k$ in time period $t$, where, $i \in W_{p}, p \in R_{o}$, $k \in H_{o}, t \in T, 0$ otherwise

$\pi_{i p t}^{o \psi} \quad=1$, if rail is selected as mode of transport for shipments starting from origin warehouse $i$ of region $p$ in time period $t$, where, $i \in W_{p}, p \in R_{o}, t \in T, 0$ otherwise

$\delta_{m j q t}^{d \phi} \quad=1$, if road is selected as mode of transport for shipping the quantity, $x_{i p k m t}^{j q}$ from destination hub $m$ to destination warehouse $j$ of region $q$ in time period $t$, where, $j \in W_{q}, q \in R_{d}, m \in H_{d}, t \in T, 0$ otherwise

$\pi_{m t}^{d \phi} \quad=1$, if road is selected as mode of transport for shipments starting from destination hub $m$ in time period $t$, where, $m \in H_{d}, t \in T, 0$ otherwise

$\delta_{m j q t}^{d \psi} \quad=1$, if rail is selected as mode of transport for shipping the quantity, $x_{i p k m t}^{j q}$ from destination hub $m$ to destination warehouse $j$ of region $q$ in time period $t$, where, $j \in W_{q}, q \in R_{d}, m \in H_{d}, t \in T, 0$ otherwise

$\pi_{m t}^{d \psi} \quad=1$, if rail is selected as mode of transport for shipments starting from destination hub $m$ in time period $t$, where, $m \in H_{d}, t \in T, 0$ otherwise 
$\delta_{k m t} \quad=1$, if there is positive shipment quantity of shipment from origin hub $k$ to destination hub $m$ in time period $t$, where, $k \in H_{o}, m \in H_{d}, t \in T, 0$ otherwise

$z_{k t} \quad=1$, if hub $k$ is open in time period $t, 0$ otherwise

$w_{m t} \quad=1$, if hub $m$ is open in time period $t, 0$ otherwise

$v_{\phi} \quad$ Truck speed $(\mathrm{km} / \mathrm{hr})$

$v_{\psi} \quad$ Train speed $(\mathrm{km} / \mathrm{hr})$

\section{Appendix B. Truck fuel consumption}

According to Demir et al., (2014), the total amount of fuel consumed ( $F$ ) by a truck carrying load $f$ to travel a fixed distance $a$ with speed $v$ is given by,

$F(v, a, f)=\frac{a \lambda}{v}\left(\mu B V+w \gamma \omega v+\gamma \omega f v+\zeta \gamma v^{3}\right)$

where, $\lambda=\frac{\xi}{\kappa \chi}$ and $\gamma=\frac{1}{1000 \eta_{t f} \eta}$ are constants. $\chi$ is the conversion factor for unit of fuel from $\mathrm{g} / \mathrm{s}$ to $1 / \mathrm{s}, \omega=\tau+g \sin \theta+g C_{r} \cos \theta$ is truck arc specific constant, where, $\tau, C_{r}$, and $\theta$ are acceleration of the truck, coefficient of rolling resistance and road angle respectively. $\zeta=\frac{C_{d} \rho A}{2}$ is the truck specific constant, where, $C_{d}, \rho$, and $A$ indicate aerodynamic drag coefficient, air density and truck frontal surface area respectively. $B, \xi, V, \mu, \eta_{t f}$, and $w$ represent engine speed, fuel-air ratio, engine displacement, engine friction factor, truck drive train efficiency and empty curb weight in kilograms respectively. $\kappa$ and $\eta$ are fuel specific constants.

\section{Appendix C. Rail fuel consumption}

Chang and Morlok, (2005) formalized the fuel energy consumed by a locomotive or a wagon in rail transport with level paths in quadratic form as a function of speed and distance as shown in Eq. (C.1), where, $z_{a}$ is fuel consumed (in $\mathrm{kWh}$ ) for traversing through arc $a, R$ is the fuel rate 
(in $\mathrm{kWh} / \mathrm{N}-\mathrm{km}$ ), $L_{a}$ is length of $\operatorname{arc} a$ in $\mathrm{km}$ and $v_{\psi}$ is train speed $(\mathrm{km} / \mathrm{hr})$. Formulae used to estimate algebraic coefficients, $u_{a}^{r}, u_{a}^{s}$, and $u_{a}^{c}$ as given by Chang and Morlok, (2005) for single wagon case are shown in Table C.1.

$$
z_{p}=R L_{p}\left(u_{p}^{r}+u_{p}^{s} v_{\psi}+u_{p}^{c} v_{\psi}^{2}\right)
$$

Table C.1 Velocity coefficients single and multiple vehicle types

\begin{tabular}{llll}
\hline Vehicle type & $u_{a}^{r}$ & $u_{a}^{s}$ & $u_{a}^{c}$ \\
\hline $\begin{array}{l}\text { Single vehicle } \\
\text { (locomotive or wagon) }\end{array}$ & $1.5\left(W_{e}+W_{\text {ship }}\right)$ & $0.03\left(W_{e}\right)$ & $C A$ \\
$\begin{array}{l}\text { Single train } \\
\text { (multiple locomotives }\end{array}$ & $1.5\left(n_{w} W_{w}+n_{l} W_{l}+W_{\text {ship }}\right)$ & $0.03\left(n_{w} W_{w}+n_{l} W_{l}+W_{\text {ship }}\right)$ & $n_{w} C_{w} A_{w}+n_{l} C_{l} A_{l}$ \\
$\begin{array}{l}\text { or wagons) } \\
\text { Multiple trains }\end{array}$ & $+18\left(n_{w}+n_{l}\right) N$ & & \\
& $1.5\left[l_{a}\left(n_{w} W_{w}+n_{l} W_{l}\right)\right.$ & $0.03\left[l_{a}\left(n_{w} W_{w}+n_{l} W_{l}\right)\right.$ & $l_{a}\left(n_{w} C_{w} A_{w}\right.$ \\
& $\left.+W_{\text {ship }}\right]+18 l_{a}\left(n_{w}+n_{l}\right) N$ & $\left.+W_{\text {ship }}\right]$ & $\left.+n_{l} C_{l} A_{l}\right)$ \\
\hline
\end{tabular}

$W_{e}=$ Empty weight of vehicle, $W_{w}=$ Empty weight of wagon, $W_{l}=$ Weight of locomotive, $W_{\text {ship }}=$ Weight of shipment, $N=$ Number of axles in vehicle, $C=$ Air drag Coefficient of vehicle, $C_{w}=$ Air drag Coefficient of wagon, $C_{l}=$ Air drag Coefficient of locomotive, $A=$ Frontal cross sectional area of vehicle, $A_{w}=$ Frontal cross sectional area of wagon,$A_{l}=$ Frontal cross sectional area of locomotive $n_{w}=$ number of wagons, $n_{l}=$ number of locomotives, $l_{a}=$ number of trains passing through arc $a$.

\section{Appendix D. Particle swarm optimization with differential evolution (PSODE)}

Amalgamating the procedures of Particle Swarm Optimization (PSO) and Differential Evolution (DE), a versatile variant of these two approaches was proposed by Liu et al. (2010) for constrained single objective optimization problems and later generalized by Epitropakis et al. (2012). In this approach, the hybrid algorithm is initialized with two different equal sized populations, pop $_{1}$ and pop $_{2}$. The particle best from pop $_{1}$ is stored in pop $_{2}$. The members of pop $_{1}$ are sorted according to constraint violations in descending order and the members of pop $_{2}$ are mapped according to their particle best values. However, both the populations evolve separately, and the position and 
velocity of $50 \%$ population of $p^{\prime} p_{1}$ are updated according to PSO procedure. Violating individuals are redirected to the feasible region by using reflection operator as shown in Eq. (D.1)

$x_{i j}^{t}= \begin{cases}0.5\left(l(j)+x_{i j}^{t}\right), & \text { if } x_{i j}^{t}<l(j) \\ 0.5\left(u(j)+x_{i j}^{t}\right), & \text { if } x_{i j}^{t}>u(j) \\ x_{i j}^{t}, & \text { otherwise }\end{cases}$

Next step includes deployment of DE procedure on $\operatorname{pop}_{2}$. For a given individual $X_{i}^{t}=$ $\left\{x_{i 1}^{t}, x_{i 2}^{t}, x_{i j}^{t}, \ldots, x_{i n}^{t}\right\}$, such that, $i \in\{1,2,3, \ldots, D\}$ of pop $_{2}$, three new offsprings based on the Eq. (D.2), (D.3), and (D.4) are generated, where, $r_{1}, r_{2}, r_{3}, r_{4}, r_{5}$ are uniformly distributed random numbers in the range $[1, D] . \chi(\chi \in[0,2])$ is an amplification factor defined according to Eq. (D.5), where, $i_{1}, i_{2}$, and $i_{3}$ are uniformly distributed random numbers in between $[1, D]$ and $i_{1} \neq i_{2} \neq i_{3}$.

$x_{i j}^{\prime t}=x_{r_{1} j}^{t}+\chi\left(x_{r_{2} j}^{t}-x_{r_{3} j}^{t}\right)$

$x_{i j}^{t}=x_{i j}^{t}+\chi\left(x_{(b e s t) j}^{t}-x_{i j}^{t}\right)+\chi\left(x_{r_{i} j}^{t}-x_{r_{2}, j}^{t}\right)$

$x_{i j}^{t}=x_{r_{1} j}^{t}+\chi\left(x_{r_{2} j}^{t}-x_{r_{3} j}^{t}\right)+\chi\left(x_{r_{4} j}^{t}-x_{r_{5} j}^{t}\right)$

$\chi_{i}=\chi_{i_{1}}+N(0,0.5)\left(\chi_{i_{2}}-\chi_{i_{3}}\right)$

Boundary violations are repaired by treating the violated individuals with Eq. (D.6), where $w_{i j}^{t}$ and $w_{i j}^{t}$ are violated and corrected offsprings respectively. Later, the selection procedure of DE is applied on the offsprings to update the particle best members of $p_{0} p_{2}$ at the end of iteration $t$ according to Eq. (D.7), where $G(X)$ function evaluates the constraint violations of candidate solution $X$. 


$$
\begin{aligned}
& w_{i j}^{t}= \begin{cases}\left.2 l(j)-w_{i j}^{t}\right), & \text { if } w_{i j}^{t}<l(j) \\
\left.2 u(j)-w_{i j}^{t}\right), & \text { if } w_{i j}^{t}>u(j) \\
w_{i j}^{t}, & \text { otherwise }\end{cases} \\
& \text { pbest }_{i}^{t+1}= \begin{cases}W_{i}^{t}, & \text { if } f\left(W_{i}^{t}\right)<f\left(\text { pbest }_{i}^{t}\right) \cap G\left(W_{i}^{t}\right) \leq G\left(\text { pbest }_{i}^{t}\right) \\
\text { pbest }_{i}^{t}, & \text { otherwise }\end{cases}
\end{aligned}
$$

The stepwise flow of PSODE algorithm for single objective problems with its pseudocode is extensively described in Liu et al. (2010). They further enumerate benefits of its adoption to a wide range of practical problems and demonstrate their ability to outclass elementary PSO and DE procedures.

\section{Appendix E. Multi-objective PSODE (MOPSODE)}

Exploiting the dexterity of PSODE in solving single objective problems, Su and Chi, (2017) extended the hybrid swarm intelligence technique to solve multi-objective problems. Higher the diversity of solutions in the pareto optimal set, better is the quality of solution to a multi-objective problem. The advantages of Multi-objective PSODE (MOPSODE) strongly aligns with the interests of computational time and solution quality. The tailoring of PSODE for solving multiobjective problems engages minor modifications and assimilation of multi-objective optimization concepts with the single objective procedure. The following sub-sections delineate certain concepts essential for clear understanding of MOPSODE algorithm.

\section{E.1 Non-dominated sorting}

A solution $\mathrm{X}$ belongs to the non-dominated set $\square$, if it strictly dominates $\mathrm{X}^{\prime}$, where $\mathrm{X}^{\prime}$ is any feasible solution to the master multi-objective problem. The distinguishing feature that differentiates the multi-objective algorithm from its single objective counterpart is the computation of non-dominated individuals. Several benchmark multi-objective algorithms accommodate sorting of non-dominated solutions after evaluating non-dominated solutions (Demir et al. 2014). 
Non-dominated sorting ensures the transfer of elite solutions in the solution space to subsequent iterations and contributes to significant reduction in computational time. Sorted individuals are stored in the pareto optimal set, $P$, where $P \subseteq \square$. NSGA-II (Deb et al., 2002) is considered as a pioneering bench mark that incorporates sorting to arrive at near optimal pareto fronts. The solution diversity among the elements of $P$ is maintained by screening the sorted individuals based on crowding distance (a metric used to measure distance between non-dominated solutions). In addition to this, MOPSODE impregnates high quality of solution diversity amongst selected non-dominated individuals, by virtue of using the hybrid technique, PSODE which retains the capability of exploring diverse set of solutions.

\section{E.2 Selection of pbest and gbest}

In PSODE, pbest and gbest iteratively capture the particle best and global best solutions respectively. According to the nature of the objective function, for minimization or maximization objectives, as the case may be, pbest and gbest in PSODE are updated as identical to PSO. However, in the case of multi-objective problems, pbest and gbest must be selected from a pool of non-dominated solutions. The choice of decision maker plays a critical role in selecting the most effective iteration best values to be carry forwarded to the next iteration. For each particle $i$ in the $t^{\text {th }}$ iteration, pbest and gbest update rules adopted by Su and Chi, (2017) are represented by Eqs. (E.1) and (E.2) respectively. The index $j$ in Eq. (E.2) represents a randomly selected nondominated individual and is defined as $j \in\left\{1,2,3, \ldots,\left|\square_{t}\right|\right\}$, where $\square_{t}$ is the set of non-dominated solutions in iteration $t$.

$$
\text { pbest }_{i}^{t}= \begin{cases}x_{i}^{t}, & \text { if } x_{i}^{t} \text { dominates pbest } \\ \text { pbest }_{i}^{t-1}, & \text { else }\end{cases}
$$


gbest $_{i}^{t}= \begin{cases}x_{i j}^{t}, & \text { if } x_{i j}^{t} \text { dominates } \text { gbest }_{i}^{t-1} \\ \text { gbest }_{i}^{t}, & \text { else }\end{cases}$

\section{E.3 Execution}

The first step of execution in MOPSODE procedure involves initialization of parameters specific to PSO and DE algorithms. In the second step, particles of the swarm are initialized randomly. However, guided initialization may be adopted to kick start the search process in the right direction. The third step involves calculation of fitness and evaluation of non-dominated individuals. In the next step, current particle is updated based on PSODE update mechanism. In the fifth step, pareto optimal set is updated based on crowding distance. If the termination criteria is met, in the last step the results are plotted and stored for further analysis. The detailed list of parameters and step wise flow diagram for the algorithm are provided in Su and Chi, (2017).

\section{Appendix F. Data}

Table F.1 Region wise warehouse distribution

\begin{tabular}{|c|c|c|c|c|c|c|}
\hline Problem set & State & $\begin{array}{l}\text { Warehouse } \\
\text { type }\end{array}$ & Region 1 & Region 2 & Region 3 & Region 4 \\
\hline \multirow[t]{4}{*}{ Small } & Origin & Hub & 1 & 1 & 1 & - \\
\hline & & Non hub & 1 & 1 & 0 & - \\
\hline & Destination & Hub & 1 & 1 & 1 & - \\
\hline & & Non hub & 0 & 1 & 1 & - \\
\hline \multirow[t]{4}{*}{ Medium } & Origin & Hub & 1 & 1 & 1 & - \\
\hline & & Non hub & 1 & 2 & 0 & - \\
\hline & Destination & $\mathrm{Hub}$ & 1 & 1 & 2 & 1 \\
\hline & & Non hub & 0 & 2 & 1 & 1 \\
\hline \multirow[t]{4}{*}{ Large } & Origin & Hub & 1 & 2 & 1 & - \\
\hline & & Non hub & 2 & 2 & 2 & - \\
\hline & Destination & Hub & 1 & 1 & 2 & 0 \\
\hline & & Non hub & 0 & 2 & 1 & 3 \\
\hline
\end{tabular}


Table F.2 Numerical description of problem parameters

\begin{tabular}{llll}
\hline Parameter & Value & Parameter & Value \\
\hline$C_{i p k}^{o \phi}$ & Rs. 4/km/MT* & $K$ & $6,500-50,000 \mathrm{MT}$ \\
$C_{i p k}^{o \psi}$ & Rs. $2.5 / \mathrm{km} / \mathrm{MT}$ & $D_{q t}$ & $2,000-20,000 \mathrm{MT}$ \\
$C_{k m}^{o d}$ & Rs. $2.5 / \mathrm{km} / \mathrm{MT}$ & $I_{i p 0}$ & $25,000-100,000 \mathrm{MT}$ \\
$C_{m j q}^{d \phi}$ & Rs. $4 / \mathrm{km} / \mathrm{MT}$ & $P_{i p t}$ & $10,000-50,000 \mathrm{MT}$ \\
$C_{m j q}^{d \psi}$ & Rs. $2.5 / \mathrm{km} / \mathrm{MT}$ & $b_{o}$ & $3-5$ \\
$A_{k t}^{o}$ & Rs. $5 \times 10^{5}-$ Rs. $10 \times 10^{5}$ & $b_{d}$ & $3-5$ \\
$A_{m t}^{d}$ & Rs. $8 \times 10^{5}-\mathrm{Rs.} 12 \times 10^{5}$ & $U_{k}$ & $2 \times 10^{5} \mathrm{MT}-4 \times 10^{5} \mathrm{MT}$ \\
$C_{h}$ & Rs $7850 / \mathrm{MT}$ & $U_{m}$ & $1.5 \times 10^{5} \mathrm{MT}-3 \times 10^{5} \mathrm{MT}$ \\
$C_{p}$ & Rs 100,000 & $\mu_{o k}$ & $0.04-0.06$ \\
$a_{i p k}^{o \phi}$ & $75 \mathrm{~km}-1,000 \mathrm{~km}$ & $\mu_{d m}$ & $0.05-0.07$ \\
$a_{i p k}^{o \psi}$ & $75 \mathrm{~km}-1,000 \mathrm{~km}$ & $C_{\phi}$ & $15 \mathrm{MT}$ \\
$a_{k m}^{o d}$ & $100 \mathrm{~km}-1,500 \mathrm{~km}$ & $C_{\psi}$ & $4,000 \mathrm{MT}$ \\
$a_{m j q}^{d \phi}$ & $50 \mathrm{~km}-500 \mathrm{~km}$ & $\rho_{i p t}^{o \phi}$ & $1,000-5,000$ \\
$a_{m j q}^{d \psi}$ & $50 \mathrm{~km}-500 \mathrm{~km}$ & $\rho_{i p t}^{o \psi}$ & $10-40$ \\
$\tau_{j q t}$ & $1000-3000 \mathrm{~h}$ & $\sigma_{k t}$ & $20-45$ \\
$\alpha$ & 0.8 & $\rho_{m t}^{d \phi}$ & $1,000-5,000$ \\
$\beta$ & 0.8 & $\rho_{m t}^{d \psi}$ & $10-40$ \\
\hline & & & \\
\hline & & & \\
\hline
\end{tabular}

Table F.3 Food grain wastage threshold levels for sensitivity analysis

\begin{tabular}{lll}
\hline Level & $\begin{array}{l}\text { Percentage decrease in wastage } \\
\text { threshold }\end{array}$ & Food grain wastage threshold $(K), \mathrm{MT}^{*}$ \\
\hline 1 & 0 & 450,000 \\
2 & 30 & 315,000 \\
3 & 50 & 225,000 \\
4 & 70 & 135,000 \\
5 & 80 & 90,000
\end{tabular}

${ }^{*}$ MT - Metric ton 
Table F.4 Intermodal hub capacity levels for sensitivity analysis

\begin{tabular}{lll}
\hline Level & Origin intermodal hub capacity, MT & Destination intermodal hub capacity, MT \\
\hline 1 & 400,000 (Low) & 620,000 (Low) \\
2 & 850,000 (High) & 620,000 (Low) \\
3 & 400,000 (Low) & 920,000 (High) \\
4 & 850,000 (High) & 920,000 (High) \\
\hline
\end{tabular}

"MT - Metric ton

Table F.5 MOPSODE parameter setting

\begin{tabular}{ll}
\hline Parameter & Value \\
\hline Population size, $N$ & 200 \\
Number of iterations, $E$ & 200 \\
Inertia weight, $w$ & 0.9 \\
Local learning factor, $c_{1}$ & 0.1 \\
Global learning factor, $c_{2}$ & 0.98 \\
Number of objectives & 2 \\
\hline
\end{tabular}

Table F.6 NSGA II parameter setting

\begin{tabular}{ll}
\hline Parameter & Value \\
\hline Population size, $N$ & 200 \\
Number of iterations, $E$ & 200 \\
$p_{c}$ & 0.9 \\
$p_{m}$ & 0.1 \\
Number of objectives & 2 \\
\hline
\end{tabular}




\section{Appendix G. Graphical illustration}

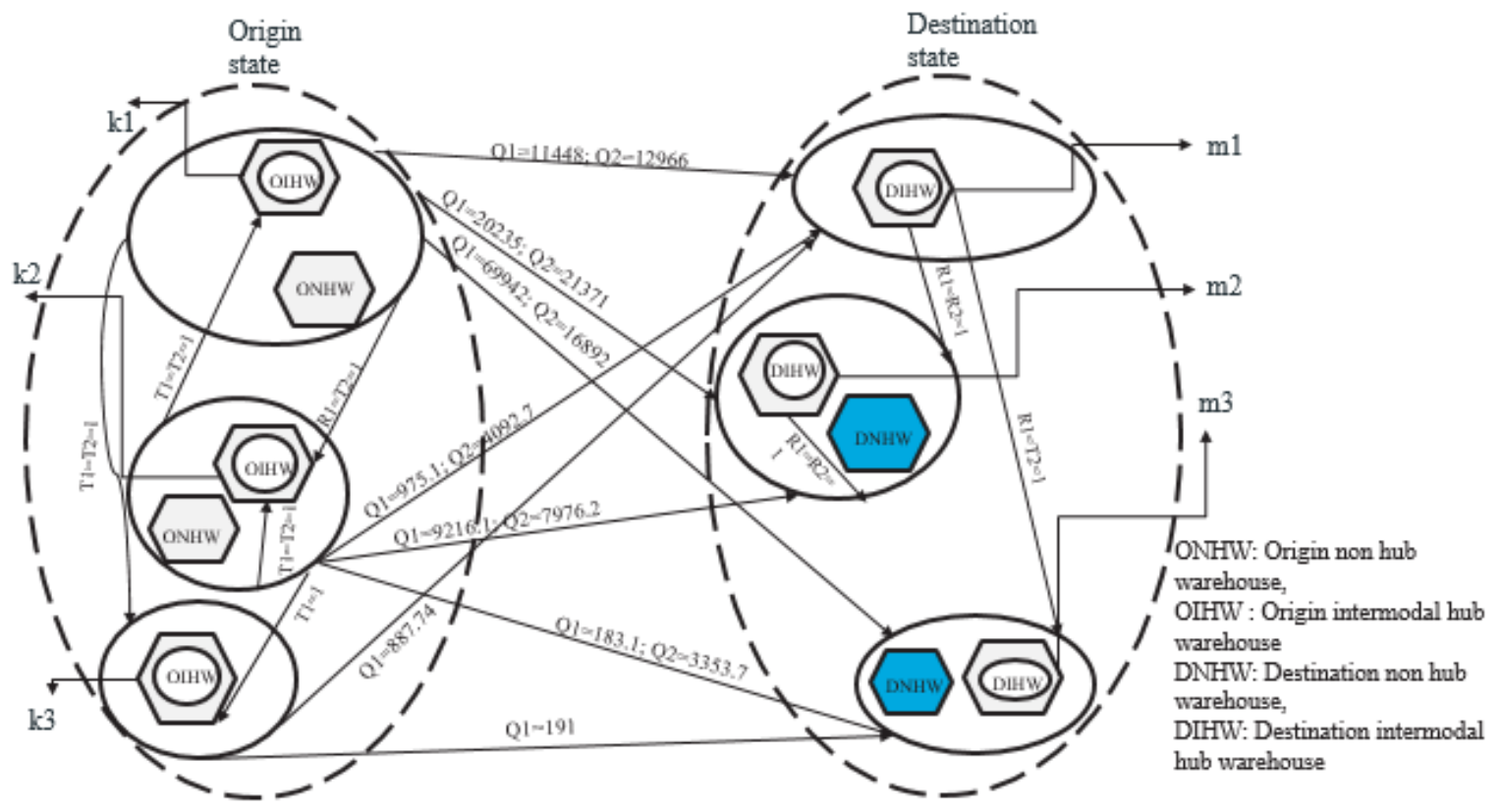

Fig. G.1 (a). Graphical illustration of sample pareto optimal shipment quantity in between states and modes selected within states for small dataset.

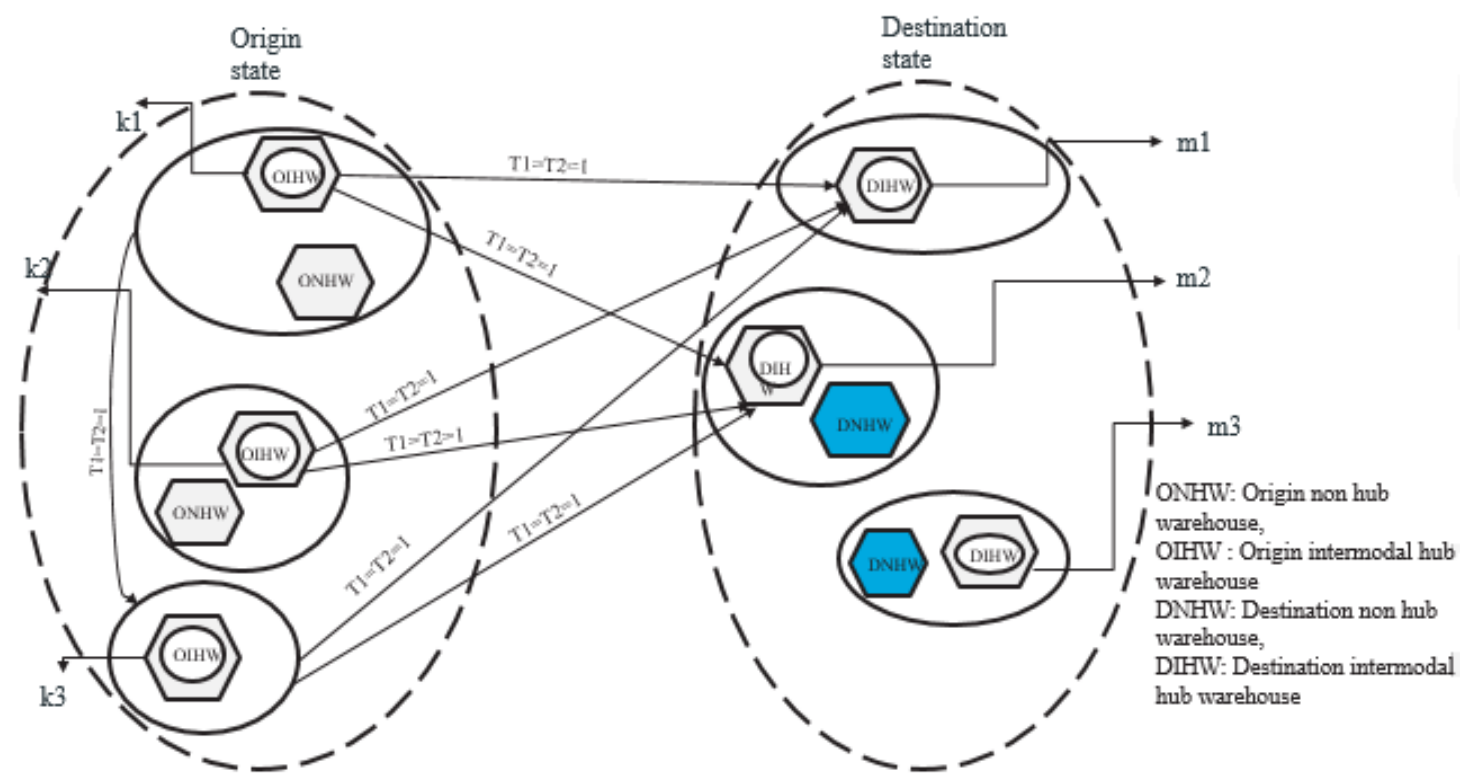

Fig. G.1 (b). Graphical illustration of sample pareto optimal rake allocation plan in between states for small dataset. 


\section{Appendix H. Decision variable result summary}

Table H.1 Region wise food grain shipment, route selection and transportation mode selection summary of sample pareto solution for all problem sets

\begin{tabular}{|c|c|c|c|c|c|c|c|c|c|c|c|c|}
\hline \multirow[t]{2}{*}{$\begin{array}{l}\text { Problem } \\
\text { set }\end{array}$} & \multirow[t]{2}{*}{$\begin{array}{l}\text { Origin } \\
\text { region } \\
(p)\end{array}$} & \multirow[t]{2}{*}{$\begin{array}{l}\text { Time } \\
\text { period } \\
(t)\end{array}$} & \multicolumn{4}{|c|}{$\begin{array}{l}\text { Food grain shipment quantity reaching region } \\
q(\mathrm{MT})\end{array}$} & \multicolumn{4}{|c|}{$\begin{array}{l}\text { No. of routes directed towards } \\
\text { region } q\end{array}$} & \multicolumn{2}{|c|}{$\begin{array}{l}\text { No. of warehouses } \\
\text { initiating road/rail } \\
\text { transport in region } p\end{array}$} \\
\hline & & & $q=1$ & $q=2$ & $q=3$ & $q=4$ & $q=1$ & $q=2$ & $q=3$ & $q=4$ & Road & Rail \\
\hline \multirow[t]{6}{*}{ Small } & \multirow[t]{2}{*}{1} & 1 & 11,448 & 20,235 & 69,942 & - & 3 & 7 & 3 & - & 1 & 2 \\
\hline & & 2 & 12,966 & 21,371 & 16,892 & - & 3 & 6 & 3 & - & 0 & 2 \\
\hline & \multirow[t]{2}{*}{2} & 1 & 975.1 & $9,216.1$ & 183.1 & - & 3 & 8 & 1 & - & 2 & 1 \\
\hline & & 2 & $4,092.7$ & $7,976.2$ & $3,353.7$ & - & 2 & 4 & 2 & - & 0 & 2 \\
\hline & \multirow[t]{2}{*}{3} & 1 & 0 & 0 & 191 & - & 0 & 0 & 1 & - & 1 & 0 \\
\hline & & 2 & 887.74 & 0 & 0 & - & 1 & 0 & 0 & - & 1 & 1 \\
\hline \multirow[t]{9}{*}{ Medium } & \multirow[t]{3}{*}{1} & 1 & 0 & 43,215 & 12,505 & 20,780 & 0 & 28 & 18 & 10 & 2 & 2 \\
\hline & & 2 & $4,429.9$ & 30,318 & 18,658 & 713 & 3 & 13 & 14 & 7 & 1 & 1 \\
\hline & & 3 & $4,829.9$ & 11,532 & 18,138 & 13,891 & 2 & 10 & 10 & 9 & 1 & 2 \\
\hline & \multirow[t]{3}{*}{2} & 1 & 0 & 15,945 & 53,978 & 5,531 & 0 & 33 & 20 & 12 & 2 & 1 \\
\hline & & 2 & 15,902 & 29,903 & 43,492 & 22,975 & 4 & 27 & 20 & 13 & 3 & 2 \\
\hline & & 3 & 27,493 & 17,545 & 66,199 & 28,183 & 6 & 32 & 20 & 19 & 3 & 3 \\
\hline & \multirow[t]{3}{*}{3} & 1 & 0 & 92.4 & $1,925.1$ & 7.96 & 0 & 7 & 5 & 3 & 1 & 1 \\
\hline & & 2 & 1.45 & $9,988.3$ & 2.5 & 123.97 & 1 & 5 & 5 & 2 & 0 & 1 \\
\hline & & 3 & 102.68 & 7,369 & 665.4 & 39.37 & 1 & 6 & 4 & 4 & 1 & 1 \\
\hline \multirow[t]{9}{*}{ Large } & \multirow[t]{3}{*}{1} & 1 & 18,936 & 34,988 & 21,784 & 7,133 & 7 & 31 & 12 & 44 & 3 & 3 \\
\hline & & 2 & 9,198 & 41,413 & 23,611 & 16,190 & 7 & 38 & 21 & 57 & 3 & 3 \\
\hline & & 3 & 37,791 & 58,053 & 26,889 & 18,577 & 4 & 24 & 13 & 28 & 3 & 1 \\
\hline & \multirow[t]{3}{*}{2} & 1 & 439.6 & 26,159 & $9,333.3$ & 6,967 & 3 & 31 & 9 & 44 & 4 & 4 \\
\hline & & 2 & 69.6 & 36,148 & $1,831.9$ & 30,214 & 7 & 52 & 23 & 66 & 4 & 4 \\
\hline & & 3 & $9,415.4$ & 18,509 & $8,335.3$ & 11,548 & 8 & 46 & 26 & 54 & 4 & 4 \\
\hline & \multirow[t]{3}{*}{3} & 1 & 2,675 & 9,961 & 1,127 & 15,744 & 7 & 34 & 9 & 40 & 2 & 3 \\
\hline & & 2 & 23,189 & 6,744 & 32,988 & 11,730 & 5 & 39 & 19 & 51 & 3 & 2 \\
\hline & & 3 & 20,932 & 20,372 & 21,402 & 21,098 & 6 & 34 & 17 & 44 & 3 & 2 \\
\hline
\end{tabular}


Table H.2 Region wise summary of transportation mode selection directed towards hub $k$ for all problem sets

\begin{tabular}{|c|c|c|c|c|c|c|c|c|c|c|c|c|c|c|}
\hline \multirow{2}{*}{$\begin{array}{l}\text { Problem } \\
\text { set }\end{array}$} & \multirow{2}{*}{$\begin{array}{l}\text { Origin } \\
\text { region } \\
(p)\end{array}$} & \multirow{2}{*}{$\begin{array}{l}\text { Time- } \\
\text { period } \\
(t)\end{array}$} & \multicolumn{4}{|c|}{ No. of road routes } & \multicolumn{4}{|c|}{ No. of rail routes } & \multicolumn{4}{|c|}{ No. of trucks (rakes) } \\
\hline & & & $k=1$ & $k=2$ & $k=3$ & $k=4$ & $k=1$ & $k=2$ & $k=3$ & $k=4$ & $k=1$ & $k=2$ & $k=3$ & $k=4$ \\
\hline \multirow[t]{6}{*}{ Small } & \multirow[t]{2}{*}{1} & 1 & 0 & 1 & 0 & - & 0 & 0 & 1 & - & $0(0)$ & $1,848(0)$ & $0(3)$ & - \\
\hline & & 2 & 0 & 0 & 0 & - & 0 & 1 & 1 & - & $0(0)$ & $0(6)$ & $0(6)$ & - \\
\hline & \multirow[t]{2}{*}{2} & 1 & 0 & 1 & 0 & - & 1 & 0 & 1 & - & $0(1)$ & $56(0)$ & $0(2)$ & - \\
\hline & & 2 & 0 & 0 & 0 & - & 1 & 1 & 0 & - & $0(2)$ & $0(3)$ & $0(0)$ & - \\
\hline & \multirow[t]{2}{*}{3} & 1 & 0 & 0 & 0 & - & 0 & 0 & 0 & - & $0(0)$ & $0(0)$ & $0(0)$ & - \\
\hline & & 2 & 0 & 0 & 0 & - & 0 & 0 & 0 & - & $0(0)$ & $0(0)$ & $0(0)$ & - \\
\hline \multirow[t]{9}{*}{ Medium } & \multirow[t]{3}{*}{1} & 1 & 0 & 1 & 0 & - & 1 & 0 & 1 & - & $0(10)$ & $1,055(0)$ & $0(6)$ & - \\
\hline & & 2 & 0 & 1 & 1 & - & 0 & 0 & 0 & - & $0(0)$ & $396(0)$ & $3,108(0)$ & - \\
\hline & & 3 & 0 & 0 & 0 & - & 1 & 0 & 1 & - & $0(2)$ & $0(0)$ & $0(6)$ & - \\
\hline & \multirow[t]{3}{*}{2} & 1 & 1 & 1 & 0 & - & 1 & 1 & 1 & - & $478(12)$ & $18(1)$ & $0(6)$ & - \\
\hline & & 2 & 1 & 1 & 1 & - & 0 & 1 & 0 & - & $3,260(0)$ & $1,346(1)$ & $341(0)$ & - \\
\hline & & 3 & 1 & 0 & 1 & - & 1 & 2 & 1 & - & $2,228(4)$ & $0(16)$ & $1,066(1)$ & - \\
\hline & \multirow[t]{3}{*}{3} & 1 & 0 & 0 & 0 & - & 0 & 0 & 0 & - & $0(0)$ & $0(0)$ & $0(0)$ & - \\
\hline & & 2 & 0 & 0 & 0 & - & 0 & 0 & 0 & - & $0(0)$ & $0(0)$ & $0(0)$ & - \\
\hline & & 3 & 0 & 0 & 0 & - & 0 & 0 & 0 & - & $0(0)$ & $0(0)$ & $0(0)$ & - \\
\hline \multirow[t]{9}{*}{ Large } & \multirow[t]{3}{*}{1} & 1 & 0 & 0 & 2 & 1 & 1 & 1 & 0 & 1 & $0(1)$ & $0(6)$ & $558(0)$ & $2,821(2)$ \\
\hline & & 2 & 0 & 2 & 1 & 1 & 1 & 0 & 1 & 1 & $0(2)$ & $970(0)$ & $435(6)$ & $481(9)$ \\
\hline & & 3 & 0 & 0 & 0 & 2 & 0 & 1 & 1 & 0 & $0(0)$ & $0(10)$ & $0(2)$ & $4,775(0)$ \\
\hline & \multirow[t]{3}{*}{2} & 1 & 0 & 1 & 1 & 0 & 1 & 0 & 1 & 1 & $0(1)$ & $1,379(0)$ & $148(2)$ & $0(3)$ \\
\hline & & 2 & 0 & 2 & 1 & 2 & 1 & 0 & 1 & 0 & $0(1)$ & $1,105(0)$ & $572(11)$ & $9(0)$ \\
\hline & & 3 & 1 & 1 & 1 & 1 & 1 & 1 & 0 & 1 & $597(2)$ & $1,418(1)$ & $59(0)$ & $100(1)$ \\
\hline & \multirow[t]{3}{*}{3} & 1 & 1 & 0 & 0 & 0 & 1 & 1 & 2 & 1 & $23(2)$ & $0(2)$ & $0(2)$ & $0(1)$ \\
\hline & & 2 & 1 & 1 & 1 & 2 & 1 & 1 & 0 & 1 & $48(4)$ & $1,834(1)$ & $349(0)$ & $15(7)$ \\
\hline & & 3 & 1 & 1 & 0 & 1 & 1 & 0 & 1 & 0 & $140(14)$ & $161(0)$ & $0(1)$ & $1,464(0)$ \\
\hline
\end{tabular}


Table H.3 Region wise summary transportation mode selection in destination state for small and medium problem sets

\begin{tabular}{|c|c|c|c|c|c|c|c|c|c|c|c|c|c|c|c|c|}
\hline \multirow[t]{2}{*}{$\begin{array}{l}\text { Problem } \\
\text { set }\end{array}$} & \multirow[t]{2}{*}{$\begin{array}{l}\text { D-hub } \\
(m)\end{array}$} & \multirow[t]{2}{*}{$\begin{array}{l}\text { Time- } \\
\text { period } \\
(t)\end{array}$} & \multicolumn{4}{|c|}{$\begin{array}{l}\text { No. of road routes directed } \\
\text { towards region } q\end{array}$} & \multicolumn{4}{|c|}{$\begin{array}{l}\text { No. of rail routes directed } \\
\text { towards region } q\end{array}$} & \multicolumn{4}{|c|}{$\begin{array}{l}\text { No. of trucks (rakes) directed } \\
\text { towards region } q\end{array}$} & \multicolumn{2}{|c|}{$\begin{array}{l}\text { Destination hubs } \\
\text { initiating } \\
\text { road/rail transport }\end{array}$} \\
\hline & & & $q=1$ & $q=2$ & $q=3$ & $q=4$ & $q=1$ & $q=2$ & $q=3$ & $q=4$ & $q=1$ & $q=2$ & $q=3$ & $q=4$ & Road & Rail \\
\hline \multirow[t]{6}{*}{ Small } & \multirow[t]{2}{*}{1} & 1 & 0 & 1 & 1 & - & 0 & 0 & 0 & - & $0(0)$ & $147(0)$ & $462(0)$ & - & 1 & 0 \\
\hline & & 2 & 0 & 1 & 0 & - & 0 & 0 & 1 & - & $0(0)$ & $0(0)$ & $0(5)$ & - & 1 & 1 \\
\hline & \multirow[t]{2}{*}{2} & 1 & 0 & 1 & 0 & - & 0 & 0 & 0 & - & $0(0)$ & $908(0)$ & $0(0)$ & - & 1 & 0 \\
\hline & & 2 & 0 & 1 & 0 & - & 0 & 0 & 0 & - & $0(0)$ & $902(0)$ & $0(0)$ & - & 1 & 0 \\
\hline & \multirow[t]{2}{*}{3} & 1 & 0 & 0 & 0 & - & 0 & 0 & 0 & - & $0(0)$ & $0(0)$ & $0(0)$ & - & 0 & 0 \\
\hline & & 2 & 0 & 0 & 0 & - & 0 & 0 & 0 & - & $0(0)$ & $0(0)$ & $0(0)$ & - & 0 & 0 \\
\hline \multirow[t]{15}{*}{ Medium } & \multirow[t]{3}{*}{1} & 1 & 0 & 0 & 0 & 0 & 0 & 0 & 0 & 0 & $0(0)$ & $0(0)$ & $0(0)$ & $0(0)$ & 0 & 0 \\
\hline & & 2 & 0 & 1 & 0 & 1 & 0 & 0 & 1 & 0 & $0(0)$ & $1038(0)$ & $0(2)$ & $6(0)$ & 1 & 1 \\
\hline & & 3 & 0 & 0 & 1 & 1 & 0 & 1 & 0 & 0 & $0(0)$ & $0(1)$ & $61(0)$ & $948(0)$ & 1 & 1 \\
\hline & \multirow[t]{3}{*}{2} & 1 & 0 & 0 & 1 & 1 & 0 & 1 & 0 & 0 & $0(0)$ & $0(2)$ & $8(0)$ & $215(0)$ & 1 & 1 \\
\hline & & 2 & 0 & 0 & 1 & 0 & 0 & 2 & 0 & 1 & $0(0)$ & $0(6)$ & $952(0)$ & $0(4)$ & 1 & 1 \\
\hline & & 3 & 0 & 0 & 0 & 0 & 0 & 0 & 0 & 0 & $0(0)$ & $0(0)$ & $0(0)$ & $0(0)$ & 0 & 0 \\
\hline & \multirow[t]{3}{*}{3} & 1 & 0 & 0 & 0 & 0 & 0 & 2 & 0 & 1 & $0(0)$ & $0(3)$ & $0(0)$ & $0(1)$ & 0 & 1 \\
\hline & & 2 & 0 & 0 & 1 & 0 & 0 & 1 & 0 & 0 & $0(0)$ & $0(1)$ & $611(0)$ & $0(0)$ & 1 & 1 \\
\hline & & 3 & 0 & 1 & 0 & 1 & 0 & 1 & 1 & 0 & $0(0)$ & $501(3)$ & $0(2)$ & $24(0)$ & 1 & 1 \\
\hline & \multirow[t]{3}{*}{4} & 1 & 0 & 1 & 0 & 0 & 0 & 1 & 1 & 0 & $0(0)$ & $330(1)$ & $0(4)$ & $0(0)$ & 1 & 1 \\
\hline & & 2 & 0 & 0 & 0 & 0 & 0 & 2 & 0 & 1 & $0(0)$ & $0(2)$ & $0(0)$ & $0(2)$ & 0 & 1 \\
\hline & & 3 & 0 & 2 & 0 & 1 & 0 & 0 & 0 & 0 & $0(0)$ & $835(0)$ & $0(0)$ & $1(0)$ & 1 & 0 \\
\hline & \multirow[t]{3}{*}{5} & 1 & 0 & 1 & 0 & 0 & 0 & 1 & 1 & 0 & $0(0)$ & $166(1)$ & $0(1)$ & $0(0)$ & 1 & 1 \\
\hline & & 2 & 0 & 0 & 0 & 0 & 0 & 0 & 0 & 0 & $0(0)$ & $0(0)$ & $0(0)$ & $0(0)$ & 0 & 0 \\
\hline & & 3 & 0 & 1 & 0 & 1 & 0 & 0 & 0 & 0 & $0(0)$ & $44(0)$ & $0(0)$ & $1(0)$ & 1 & 0 \\
\hline
\end{tabular}


Table H.4 Region wise summary of transportation mode selection in destination state for large problem set

\begin{tabular}{|c|c|c|c|c|c|c|c|c|c|c|c|c|c|c|c|}
\hline \multirow[t]{2}{*}{$\begin{array}{l}\text { D-hub } \\
(m)\end{array}$} & \multirow[t]{2}{*}{$\begin{array}{l}\text { Time- } \\
\text { period } \\
(t)\end{array}$} & \multicolumn{4}{|c|}{$\begin{array}{l}\text { No. of road routes directed } \\
\text { towards region } q\end{array}$} & \multicolumn{4}{|c|}{$\begin{array}{l}\text { No. of rail routes directed } \\
\text { towards region } q\end{array}$} & \multicolumn{4}{|c|}{$\begin{array}{l}\text { No. of rakes or trucks directed towards } \\
\text { region } q\end{array}$} & \multicolumn{2}{|c|}{$\begin{array}{l}\text { No. of } \\
\text { destination hubs } \\
\text { initiating } \\
\text { road/rail } \\
\text { transport }\end{array}$} \\
\hline & & $q=1$ & $q=2$ & $q=3$ & $q=4$ & $q=1$ & $q=2$ & $q=3$ & $q=4$ & $q=1$ & $q=2$ & $q=3$ & $q=4$ & Road & Rail \\
\hline \multirow[t]{3}{*}{1} & 1 & 0 & 0 & 0 & 2 & 0 & 2 & 0 & 1 & $0(0)$ & $0(3)$ & $0(0)$ & $247(2)$ & 1 & 1 \\
\hline & 2 & 0 & 2 & 0 & 3 & 0 & 0 & 1 & 0 & $0(0)$ & $39(0)$ & $0(4)$ & $1,111(0)$ & 1 & 1 \\
\hline & 3 & 0 & 1 & 0 & 1 & 0 & 1 & 1 & 2 & $0(0)$ & $229(2)$ & $0(3)$ & $1,347(5)$ & 1 & 1 \\
\hline \multirow[t]{3}{*}{2} & 1 & 0 & 0 & 0 & 2 & 0 & 2 & 0 & 1 & $0(0)$ & $0(6)$ & $0(0)$ & 779 (1) & 1 & 1 \\
\hline & 2 & 0 & 1 & 0 & 1 & 0 & 1 & 0 & 2 & $0(0)$ & $1,681(7)$ & $0(0)$ & $272(5)$ & 1 & 1 \\
\hline & 3 & 0 & 0 & 1 & 3 & 0 & 2 & 0 & 0 & $0(0)$ & $0(6)$ & $35(0)$ & $621(0)$ & 1 & 1 \\
\hline \multirow[t]{3}{*}{3} & 1 & 0 & 0 & 0 & 1 & 0 & 1 & 1 & 1 & $0(0)$ & $0(2)$ & $0(3)$ & $120(1)$ & 1 & 1 \\
\hline & 2 & 0 & 0 & 0 & 0 & 0 & 0 & 0 & 0 & $0(0)$ & $0(0)$ & $0(0)$ & $0(0)$ & 0 & 0 \\
\hline & 3 & 0 & 0 & 0 & 0 & 0 & 0 & 0 & 0 & $0(0)$ & $0(0)$ & $0(0)$ & $0(0)$ & 0 & 0 \\
\hline \multirow[t]{3}{*}{4} & 1 & 0 & 0 & 0 & 0 & 0 & 0 & 0 & 0 & $0(0)$ & $0(0)$ & $0(0)$ & $0(0)$ & 0 & 0 \\
\hline & 2 & 0 & 1 & 1 & 0 & 0 & 1 & 0 & 3 & $0(0)$ & 104 (1) & $740(0)$ & $0(6)$ & 1 & 1 \\
\hline & 3 & 0 & 1 & 1 & 1 & 0 & 1 & 0 & 2 & $0(0)$ & $8(1)$ & $258(0)$ & $1(2)$ & 1 & 1 \\
\hline
\end{tabular}


Table H.5 Inter-state transport rake allocation and destination hub location summary of sample pareto solution for all problem sets

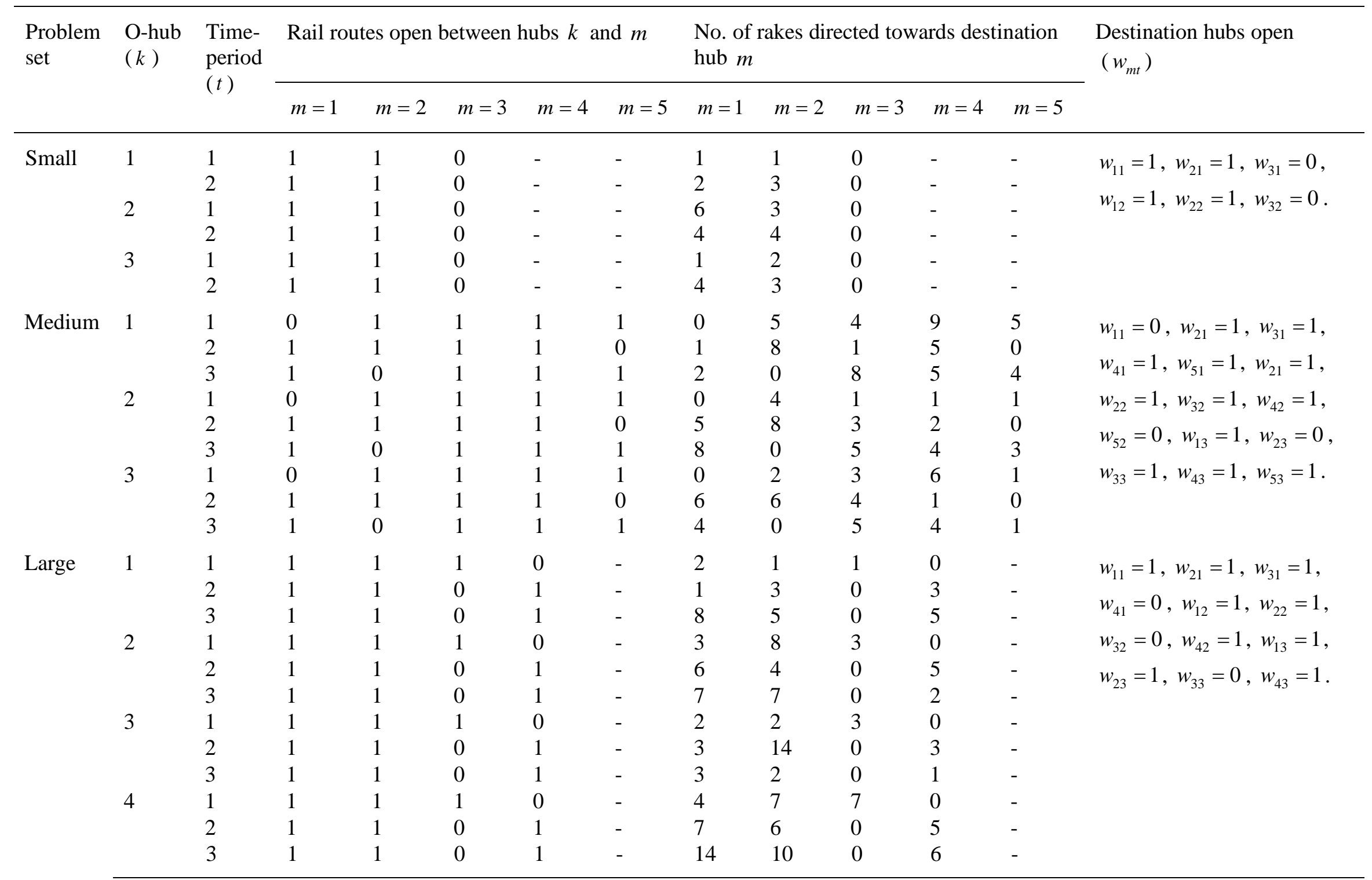




\section{References}

Asgari, N., Farahani, R. Z., Rashidi-Bajgan, H., Sajadieh, M. S. 2013. Developing model-based software to optimise wheat storage and transportation: A real-world application. Applied Soft Computing, 13(2), 1074-1084.

Centobelli, P., Cerchione, R., Esposito, E., 2017. Environmental sustainability in the service industry of transportation and logistics service providers: Systematic literature review and research directions. Transport. Res. Part D: Transp. Environ. 53, 454-470.

Chang, D. J., Morlok, E. K., 2005. Vehicle speed profiles to minimize work and fuel consumption. J. Transport. Eng. 131 (3), 173-182.

Chávez, M.M.M., Sarache, W. and Costa, Y., 2018. Towards a comprehensive modbjael of a biofuel supply chain optimization from coffee crop residues. Transportation Research Part E: Logistics and Transportation Review, 116, 136-162.

De, A., Kumar, S. K., Gunasekaran, A., Tiwari, M. K., 2017. Sustainable maritime inventory routing problem with time window constraints. Engineering Applications of Artificial Intelligence. 61, 77-95.

Deb, K., Pratab, S., Agarwal, S., Meyarivan, T., 2002. A Fast and Elitist Multiobjective Genetic Algorithm: NGSA-II. IEEE Trans. Evol. Comput. 6, 182-197.

Demir, E., Bektas, T., Laporte, G., 2014. The bi-objective pollution-routing problem. Eur. J. Oper. Res., 232 (3), 464-478.

Hernández-Domínguez, J. S., Toscano-Pulido, G., Coello, C. A. C. 2011. A multi-objective particle swarm optimizer enhanced with a differential evolution scheme. In International 
Conference on Artificial Evolution (Evolution Artificielle), Springer, Berlin, Heidelberg, 169-180.

Epitropakis, M.G., Plagianakos, V.P., Vrahatis, M.N., 2012. Evolving cognitive and social experience in particle swarm optimization through differential evolution: A hybrid approach. Inform. Sci. 216, 50-92.

FAO, 2011. Global Food Losses and Food Waste. Extent, Causes and Prevention.

Fattahi, M. and Govindan, K., 2018. A multi-stage stochastic program for the sustainable design of biofuel supply chain networks under biomass supply uncertainty and disruption risk: A real-life case study. Transportation Research Part E: Logistics and Transportation Review, 118, 534-567.

Garnett, T., 2011. Where are the best opportunities for reducing greenhouse gas emissions in the food system (including the food chain)? Food Policy, 36 (1), S23-S32.

Harris, I., Naim, M., Palmer, A., Potter, A., Mumford, C., 2011. Assessing the impact of cost optimization based on infrastructure modelling on $\mathrm{CO} 2$ emissions. International Journal of Production Economics, 131(1), 313-321.

Hanne, T., 1999. On the convergence of multiobjective evolutionary algorithms. European Journal of Operational Research, 117(3), 553-564.

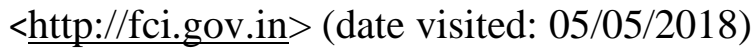

<http://pdsportal.nic.in/main.aspx > (date visited: 20/05/2018)

$<$ https://www.fois.indianrail.gov.in/foisweb/view/qry/TQ_FrgtCalcIN.jsp>(date visited: 01/05/2018)

INCCA, 2010. India: Greenhouse Gas Emissions 2007. Indian Network for Climate Change Assessment (INCCA), The Ministry of Environment \& Forests, Government of India, 63. 
IPCC, 2014 Fifth Assessment Report. Chapter 8: Transportation.

Ishfaq, R., Sox, C.R., 2011. Hub location-allocation in intermodal logistic networks. Eur. J. Oper. Res. 210, 213-230.

Kumar, R. S., Kondapaneni, K., Dixit, V., Goswami, A., Thakur, L. S., Tiwari, M. K., 2016. Multiobjective modeling of production and pollution routing problem with time window: A selflearning particle swarm optimization approach. Comput. Ind. Eng. 99, 29-40.

Lee, D. H., Dong, M., Bian, W. 2010. The design of sustainable logistics network under uncertainty. International Journal of Production Economics, 128(1), 159-166.

Liotta, G., Stecca, G., Kaihara, T., 2015. Optimisation of freight flows and sourcing in sustainable production and transportation networks. Int. J. Prod. Econ. 164, 351-365.

Li, X., Li, M., 2015. Multiobjective local search algorithm-based decomposition for multiobjective permutation flow shop scheduling problem. IEEE Transactions on Engineering Management, 62(4), 544-557.

Liu, H., Cai, Z., Wang, Y., 2010. Hybridizing particle swarm optimization with differential evolution for constrained numerical and engineering optimization. Appl. Soft. Comput. 10, 629-640.

Maiyar, L.M., Thakkar, J.J., 2017. A combined tactical and operational deterministic food grain transportation model: particle swarm based optimization approach. Comput. Ind. Eng. 110, $30-42$.

Maiyar, L.M., Thakkar, J.J., 2018. Modelling and analysis of inter-modal food grain transportation under hub disruption towards sustainability. Int. J. Prod. Econ. In press. 
Meng, Q., Wang, X., 2011. Intermodal hub-and-spoke network design: Incorporating multiple stakeholders and multi-type containers. Transportation Research Part B: Methodological, 45(4), 724-742.

Mogale, D. G., Dolgui, A., Kandhway, R., Kumar, S. K., Tiwari, M. K., 2017. A multi-period inventory transportation model for tactical planning of food grain supply chain. Comput. Ind. Eng. 110, 379-394.

Mogale, D. G., Kumar, M., Kumar, S. K., Tiwari, M. K., 2018. Grain silo location-allocation problem with dwell time for optimization of food grain supply chain network. Transport. Res. Part E: Logist. Transport. Rev. 111, 40-69.

Niknamfar, A. H., Niaki, S. T. A., 2016. Fair profit contract for a carrier collaboration framework in a green hub network under soft time-windows: Dual lexicographic max-min approach. Transport. Res. Part E: Logist. Transport. Rev. 91, 129-151.

O’Donnell, B., Goodchild, A., Cooper, J., Ozawa, T. 2009. The relative contribution of transportation to supply chain greenhouse gas emissions: A case study of American wheat. Transport. Res. Part D: Transp. Environ. 14 (7), 487-492.

Olivier, J.G.J, Schure, K.M., Peters, J.A.H.W., 2017. Trends in global $\mathrm{CO}_{2}$ and total greenhouse gas emissions. Netherlands Environmental Assessment Agency.

Parikh, J., Panda, M., Ganesh-Kumar, A., Singh, V. 2009. $\mathrm{CO}_{2}$ emissions structure of Indian economy. Energy 34 (8), 1024-1031.

Pathak, H., Jain, N., Bhatia, A., Patel, J., Aggarwal, P. K. 2010. Carbon footprints of Indian food items. Agri. Eco. Environ. 139 (1), 66-73. 
Report of the Comptroller and Auditor General of India on Storage Management and Movement of Food Grains in Food Corporation of India. 2013. Union Government Ministry of Consumer Affairs, Food and Public Distribution.

Su, Y. X, Chi, R., 2017. Multi-objective particle swarm-differential evolution algorithm. Neural Comput. Appl., 28, 407-418.

Saberi, S., 2018. Sustainable, multiperiod supply chain network model with freight carrier through reduction in pollution stock. Transportation Research Part E: Logistics and Transportation Review, 118, 421-444.

Sahebjamnia, N., Fard, A.M.F., Hajiaghaei-Keshteli, M., 2018. Sustainable tire closed-loop supply chain network design: Hybrid metaheuristic algorithms for large-scale networks. Journal of Cleaner Production.

Sundarakani, B., Souza, R. D., Goh, M., Wagner, S. M., Manikandan, S., 2010. Modeling carbon footprints across the supply chain. Int. J. Prod. Econ. 128 (1), 43-50.

Vahdani, B., Zandieh, M., 2010. Scheduling trucks in cross-docking systems: Robust metaheuristics. Computers \& Industrial Engineering, 58(1), 12-24.

van der Vorst, J. G., Tromp, S. O., Zee, D. J. V. D. 2009. Simulation modelling for food supply chain redesign; integrated decision making on product quality, sustainability and logistics. International Journal of Production Research. 47(23), 6611-6631.

Velázquez-Martínez, J., Fransoo, J. C., Blanco, E. E., Valenzuela-Ocaña, K. B., 2016. A new statistical method of assigning vehicles to delivery areas for $\mathrm{CO}_{2}$ emissions reduction. Transport. Res. Part D: Transp. Environ. 43, 133-144. 
Wakeland, W., Cholette, S., Venkat, K. 2012. Food transportation issues and reducing carbon footprint. In Green Tech. Food Prod. Process. 211-236. Springer, US.

Wang, F., Lai, X., Shi, N. 2011. A multi-objective optimization for green supply chain network design. Decision Support Systems. 51(2), 262-269.

Zahiri, B., Zhuang, J., Mohammadi, M. 2017. Toward an integrated sustainable-resilient supply chain: A pharmaceutical case study. Transportation Research Part E: Logistics and Transportation Review. 103, 109-142.

Zhalechian, M., Tavakkoli-Moghaddam, R., Zahiri, B., Mohammadi, M. 2016. Sustainable design of a closed-loop location-routing-inventory supply chain network under mixed uncertainty. Transportation Research Part E: Logistics and Transportation Review. 89, $182-214$. 